\title{
Galileon scalar electrodynamics
}

\author{
Ashu Kushwaha ${ }^{*}$ and S. Shankaranarayanan $\odot^{\dagger}$ \\ Department of Physics, Indian Institute of Technology Bombay, Mumbai 400076, India
}

(Received 18 December 2019; accepted 27 February 2020; published 17 March 2020)

\begin{abstract}
We construct a consistent model of Galileon scalar electrodynamics. The model satisfies three essential requirements: (i) The action contains higher-order derivative terms and obeys the Galilean symmetry, (ii) equations of motion also satisfy Galilean symmetry and contain only up to second-order derivative terms in the matter fields and, hence, do not suffer from instability, and (iii) local $U(1)$ gauge invariance is preserved. We show that the nonminimal coupling terms in our model are different from that of the real scalar Galileon models; however, they match with the Galileon real scalar field action. We show that the model can lead to an accelerated expansion in the early Universe. We discuss the implications of the model for cosmological inflation.
\end{abstract}

DOI: 10.1103/PhysRevD.101.065008

\section{INTRODUCTION}

The standard relativistic field theories describing physical phenomena contain the second-order time and spatial derivatives. The perturbative approach of these field theories is highly successful in explaining the experiments and observations. However, these field theories have ultraviolet divergences, and higher-derivative terms were introduced in an attempt to remove the ultraviolet divergence [1-3].

The higher-derivative field theories are known to have Ostrogradsky instability [4]. This is because the Hamiltonian contains a term proportional to the momenta, thus leading to an unbounded Hamiltonian from below [5]. The instability itself is not a concern if the energy is an integral of motion or the higher-derivative field (say, $\pi$ ) does not interact with any other field (say, $\phi$ ) whose Hamiltonian is bounded from below. The interaction between $\pi$ and $\phi$ will pump out energy from $\pi$, leading to a runaway situation. Hence, these negative energy states can be traded by negative norm states (or ghosts), leading to nonunitary theories [6] and, therefore, unsuitable to describe physical phenomena.

Nonetheless, higher-derivative theories have some salient features that make them indispensable to understand the high-energy behavior of a theory [7]. Specifically, the divergence structure of quantum fields is expected to improve when higher-derivative terms are taken into

\footnotetext{
*ashu712@iitb.ac.in

†shanki@phy.iitb.ac.in
}

Published by the American Physical Society under the terms of the Creative Commons Attribution 4.0 International license. Further distribution of this work must maintain attribution to the author(s) and the published article's title, journal citation, and DOI. Funded by SCOAP ${ }^{3}$. account $[6,7]$. This also leads to improved convergence of Feynman diagrams. Although Einstein's gravity is not renormalizable, conformal gravity is $[8,9]$.

There is a resurgence of interest in higher-derivative theories primarily from the modifications of gravity in the short and long distances [10-15]. It is now known that higher-derivative gravity theories like $f(R)$ are degenerate, and do not suffer from Ostrogradsky instability [16]. A consistent scheme has been implemented where the Ostrogradsky instability is suppressed by requiring that the equations of motion are second order [17]. Starting from the following general action:

$L=L\left(g_{i j} ; g_{i j, i_{1}} ; \ldots ; g_{i j, i_{1} \cdots i_{p}} ; \phi ; \phi ; \phi,,_{i_{1}} ; \ldots ; \phi_{, i_{1}} \ldots i_{q}\right)$,

Horndeski showed that it is possible to obtain a secondorder equation for $\phi$ even if the action contains higher-order derivatives of the scalar field [17]. It was recently shown that the field equations in a subclass of Horndeski models have Galilean shift symmetry in flat space, i.e.,

$$
\phi \rightarrow \phi+a_{\mu} x^{\mu}+b
$$

where $a_{\mu}$ and $b$ are infinitesimal four-vector (shift parameter) and a constant scalar respectively [18,19]. Galileon scalar fields are the most general noncanonical and nonminimally coupled single-field model, which yields secondorder equations.

These theories, while naively look like higher-derivative theories, are healthy non-higher-derivative theories; their equations of motion are second order in time derivatives and do not suffer from the instability $[18,19]$. This is achieved by the addition of structure in the Lagrangianusually by a subtle cancellation of higher-derivative terms in the equations of motion, much like Lanczos-Lovelock 
theories of gravity [20-23]. Like Galileon scalars, these theories of gravity are exceptional in that the resulting equations of motion are no more than the second order. They are also free of ghosts when expanded about the flat space-time.

Recently, a vector Galileon field was constructed in a curved space-time [24]. It was shown that the electromagnetic action breaks conformal invariance and has the following three properties: model is described by vector potential $A_{\mu}$ and its derivatives, Gauge invariance is preserved, and equations of motion are linear in second derivatives of the vector potential. This is an essential result as earlier it was proven that such an action could not be constructed in flat space-time [19].

While the scalar and vector Galileon fields have been investigated, there has been no study on the interaction between the scalar and vector Galileons preserving gauge invariance. (In Ref. [25], Heisenberg et al. studied scalarvector Galileon models that do not preserve gauge invariance.) In this work, we bridge this gap by constructing a higher-derivative action of interacting fields (complex scalar Galileons interacting with a vector field), which do not lead to ghosts. We refer to this model as Galileon scalar electrodynamics. We construct a complex scalar Galileon action in flat space-time by demanding the following conditions: action must satisfy the Galileon symmetry, equations of motion must be second order, gauge invariance is preserved. Naturally, the new action has the standard scalar electrodynamics with new terms that contribute at high energies. We apply this model in the early Universe.

The rest of the manuscript is organized as follows: In Sec. II, we explicitly construct the Galileon scalar electrodynamics in flat space-time. We show that the action is invariant under the local gauge invariance in flat space-time. In Sec. III, we extend the analysis to curved space-time. We show that the minimal coupling of the matter and gravity leads to higher-derivative terms in the equations of motion. We then include nonminimal terms to the action that will lead to subtle cancellation of terms and, hence, lead to second-order equations of motion. We then apply the model to the early Universe in Sec. IV and show that the model can lead to inflation. In Sec. V, we conclude by briefly discussing the importance of these results. Appendixes A-F contain the details of the calculations in the main text.

In this work, we use $(+,-,-,-)$ metric signature and natural units $\hbar=c=1 /\left(4 \pi \epsilon_{0}\right)=1$. We set $8 \pi G=M_{P}^{-2}$. The real Galileon scalar field is denoted by $\varphi$, complex Galileon scalar field is denoted by $\pi$, and $\square=\nabla_{\mu} \nabla^{\mu}$. An overdot denotes the derivative for cosmic time.

\section{GALILEON SCALAR ELECTRODYNAMICS IN FLAT SPACE-TIME}

In this section, we obtain the higher-derivative complex scalar field action coupled to the electromagnetic field in flat space-time. In the next subsection, we list the real scalar Lagrangians obtained by Nicolis et al. [26] that lead to healthy non-higher-derivative theories. In Sec. II B, we obtain the complex scalar Lagrangian that leads to nonhigher-derivative theories. In Sec. IIC, we couple the Galileon complex scalar with the electromagnetic field $A_{\mu}$.

\section{A. Real scalar Galileon}

As mentioned earlier, the action of the real scalar Galileons $(\varphi)$ is invariant under the Galilean transformation (2). In 4-D Minkowski space-time, Nicolis et al. [26] have shown that the following five Lagrangians are invariant under the Galilean transformation:

$$
\begin{aligned}
\mathcal{L}_{1}^{\prime}= & \varphi, \quad \mathcal{L}_{2}^{\prime}=-\frac{1}{2} \partial_{\mu} \varphi \partial^{\mu} \varphi, \\
\mathcal{L}_{3}^{\prime}= & -\frac{1}{2} \square \varphi \partial_{\mu} \varphi \partial^{\mu} \varphi, \\
\mathcal{L}_{4}^{\prime}= & -\frac{1}{4}\left[(\square \varphi)^{2} \partial_{\mu} \varphi \partial^{\mu} \varphi-2 \square \varphi \partial_{\mu} \varphi \partial^{\mu} \partial_{\nu} \varphi \partial^{\nu} \varphi-\partial_{\mu} \partial_{\nu} \varphi \partial^{\mu} \partial^{\nu} \varphi \partial_{\alpha} \varphi \partial^{\alpha} \varphi+2 \partial_{\mu} \varphi \partial^{\mu} \partial_{\nu} \varphi \partial^{\nu} \partial_{\alpha} \varphi \partial^{\alpha} \varphi\right], \\
\mathcal{L}_{5}^{\prime}= & -\frac{1}{5}\left[(\square \varphi)^{3} \partial_{\mu} \varphi \partial^{\mu} \varphi-3(\square \varphi)^{2} \partial_{\mu} \varphi \partial^{\mu} \partial_{\nu} \varphi \partial^{\nu} \varphi-3 \square \varphi \partial_{\mu} \partial^{\nu} \varphi \partial^{\mu} \partial_{\nu} \varphi \partial_{\alpha} \varphi \partial^{\alpha} \varphi+6 \square \varphi \partial_{\mu} \varphi \partial^{\mu} \partial_{\nu} \varphi \partial^{\nu} \partial_{\alpha} \varphi \partial^{\alpha} \varphi\right. \\
& \left.+2 \partial_{\mu} \partial^{\nu} \varphi \partial_{\nu} \partial^{\alpha} \varphi \partial_{\alpha} \partial^{\mu} \varphi \partial_{\lambda} \varphi \partial^{\lambda} \varphi+3 \partial_{\mu} \partial^{\nu} \varphi \partial_{\nu} \partial^{\mu} \varphi \partial_{\alpha} \varphi \partial^{\alpha} \partial_{\lambda} \varphi \partial^{\lambda} \varphi-6 \partial_{\mu} \varphi \partial^{\mu} \partial_{\nu} \varphi \partial^{\nu} \partial_{\alpha} \varphi \partial^{\alpha} \partial_{\lambda} \varphi \partial^{\lambda} \varphi\right] .
\end{aligned}
$$

We want to emphasize that the above Lagrangians are the linear combinations of Lorentz invariant terms which are added (up to total derivative term) in such a way that the action is Galilean invariant. The equations of motion are second order. In the next subsection, we use these five Lagrangians to construct the complex scalar Galileon action.

\section{B. Complex scalar Galileon}

We aim to write down the Lagrangian that satisfies the following three conditions: First, it must satisfy shift symmetry (2) on $\pi$ and the complex conjugate $\left(\pi^{*}\right)$. Second, it must be Lorentz invariant. Third, the Lagrangian must be 
real. It is easy to see that the Lagrangians with the odd number of $\pi$ 's lead to complex action and, hence, are discarded. Having $\mathcal{L}_{2}^{\prime}$ and $\mathcal{L}_{4}^{\prime}$ in hand, we start with $\mathcal{L}_{2}^{\prime}$. The complex scalar Galileon Lagrangian for this case is straightforward:

$$
\mathcal{L}_{2}^{\prime}=\frac{1}{2} \partial_{\mu} \pi \partial^{\mu} \pi \Rightarrow \mathcal{L}_{2}=\frac{1}{2} \partial_{\mu} \pi \partial^{\mu} \pi^{*}
$$

where $\pi^{*}$ is the complex conjugate. The above action is invariant under the Galilean shift symmetry (2) on $\pi$ and $\pi^{*}$. In order to avoid confusion, we have used unprimed for complex scalar Lagrangian to distinguish it from the scalar Galileon Lagrangian (primed).

Next, we consider the Lorentz invariant terms of $\mathcal{L}_{4}^{\prime}$ (with two fields $\pi$ and $\pi^{*}$ ). By symmetrizing each term in the righthand side of (3c) and ignoring the double-counting, we write $\mathcal{L}_{4}$ as a linear combination of these invariants, i.e.,

$$
\begin{aligned}
\mathcal{L}_{4}= & A_{1}\left((\square \pi)^{2} \partial_{\alpha} \pi^{*} \partial^{\alpha} \pi^{*}+\text { c.c. }\right)+\mathrm{A}_{2} \square \pi^{*} \square \pi \partial_{\alpha} \pi^{*} \partial^{\alpha} \pi+\mathrm{B}_{1}\left(\square \pi \partial_{\nu} \pi \partial^{\nu} \partial^{\alpha} \pi^{*} \partial_{\alpha} \pi^{*}+\text { c.c. }\right) \\
& +B_{2}\left(\square \pi \partial_{\nu} \pi^{*} \partial^{\nu} \partial^{\alpha} \pi \partial_{\alpha} \pi^{*}+\text { c.c. }\right)+\mathrm{C}_{1}\left(\partial_{\mu} \partial_{\nu} \pi \partial^{\mu} \partial^{\nu} \pi \partial^{\alpha} \pi^{*} \partial_{\alpha} \pi^{*}+\text { c.c. }\right)+\mathrm{C}_{2} \partial_{\mu} \partial_{\nu} \pi^{*} \partial^{\mu} \partial^{\nu} \pi \partial^{\alpha} \pi^{*} \partial_{\alpha} \pi \\
& +D_{1}\left(\partial_{\mu} \pi^{*} \partial^{\mu} \partial^{\nu} \pi \partial_{\nu} \partial_{\alpha} \pi \partial^{\alpha} \pi^{*}+\text { c.c. }\right)+\mathrm{D}_{2} \partial_{\mu} \pi^{*} \partial^{\mu} \partial^{\nu} \pi^{*} \partial_{\nu} \partial_{\alpha} \pi \partial^{\alpha} \pi+\mathrm{D}_{3} \partial_{\mu} \pi^{*} \partial^{\mu} \partial^{\nu} \pi \partial_{\nu} \partial_{\alpha} \pi^{*} \partial^{\alpha} \pi
\end{aligned}
$$

where $A_{i}, B_{i}, C_{i}, D_{i}, D_{3}(i=1,2)$ are unknown complex constants, c.c. in the parentheses denote the complex conjugate part (hence total part contributing to real) of the corresponding Lorentz invariant term and the rest of the terms are real. Hence, the above Lagrangian is a real scalar.

However, for any arbitrary constants, the Lagrangian will not be Galilean invariant. Demanding the Galilean invariance of the Lagrangian (5) (up to some total derivative) leads to the constraints on the constants. Appendix A contains detailed calculations where we show that $\mathcal{L}_{4}$ depends only on two arbitrary coefficients $A_{1}$ and $A_{2}$ and the resultant equations of motion match with Nicolis et al. [26]. In Appendix A we also explicitly show that the equation of motion for this Lagrangian is the same for any value of $A_{1}$ and $A_{2}$. This is because the action $\mathcal{L}_{4}$ is invariant under the shift symmetry (2) for any value of these arbitrary constants. For simplicity, we set $A_{1}=0$. Setting $A_{1}=0$ and $A_{2}=\omega \lambda^{-6}$ in Eq. (A4) leads to

$$
\begin{aligned}
\mathcal{L}_{4}= & \frac{\omega}{2 \lambda_{\pi}^{6}}\left[2 \square \pi^{*} \square \pi \partial_{\alpha} \pi \partial^{\alpha} \pi^{*}-2 \partial_{\mu} \partial_{\nu} \pi^{*} \partial^{\mu} \partial^{\nu} \pi \partial_{\alpha} \pi \partial^{\alpha} \pi^{*}\right. \\
& \left.+\left(\square \pi \partial_{\nu} \pi^{*} \partial^{\nu} \partial^{\alpha} \pi \partial_{\alpha} \pi^{*}-\partial^{\mu} \partial^{\nu} \pi \partial_{\nu} \partial_{\alpha} \pi \partial_{\mu} \pi^{*} \partial^{\alpha} \pi^{*}+\text { c.c. }\right)\right] .
\end{aligned}
$$

Note that $A_{2}$ has a dimension $[L]^{6}$, thus $\lambda$ has dimensions of inverse length and $\omega$ can take +1 or -1 . The value of $\omega$ will be fixed in Sec. III C by demanding that the energy density of the field is always positive. In the next section, we will show that the generalization of the above Lagrangian in curved space-time matches with Deffayet et al. for some suitable value of the coefficient $\lambda_{\pi}$ [27]. The Galilean invariant action corresponding to the above Lagrangian is given by

$$
\begin{aligned}
S_{4}= & \frac{\omega}{2 \lambda_{\pi}^{6}} \int d^{4} x\left[2 \square \pi^{*} \square \pi \partial_{\alpha} \pi \partial^{\alpha} \pi^{*}-2 \partial_{\mu} \partial_{\nu} \pi^{*} \partial^{\mu} \partial^{\nu} \pi \partial_{\alpha} \pi \partial^{\alpha} \pi^{*}\right. \\
& \left.+\left(\square \pi \partial_{\nu} \pi^{*} \partial^{\nu} \partial^{\alpha} \pi \partial_{\alpha} \pi^{*}-\partial^{\mu} \partial^{\nu} \pi \partial_{\nu} \partial_{\alpha} \pi \partial_{\mu} \pi^{*} \partial^{\alpha} \pi^{*}+\text { c.c. }\right)\right] .
\end{aligned}
$$

Equation of motion for $\pi$ corresponding to the above action is

$$
\begin{aligned}
\mathcal{E}_{4}= & \frac{\omega}{2 \lambda_{\pi}^{6}}\left[-\left(\square \pi^{*}\right)^{2} \square \pi+\square \pi \partial_{\mu} \partial_{\nu} \pi^{*} \partial^{\mu} \partial^{\nu} \pi^{*}\right. \\
& \left.-2 \partial_{\mu} \partial_{\nu} \pi^{*} \partial^{\nu} \partial^{\alpha} \pi \partial^{\mu} \partial_{\alpha} \pi^{*}+2 \square \pi^{*} \partial_{\mu} \partial_{\nu} \pi^{*} \partial^{\mu} \partial^{\nu} \pi\right] .
\end{aligned}
$$

The equations of motion contain derivatives up to second order and, hence, do not lead to extra degrees of freedom. The complex scalar Galileon action in 4D Minkowski space-time is given by

$$
S_{\text {flat }}=S_{2}+S_{4},
$$

where $S_{4}$ is given by (7) and

$$
S_{2}=\frac{1}{2} \int d^{4} x \partial_{\mu} \pi \partial^{\mu} \pi^{*} .
$$

Thus, we have constructed a complex Galileon field action. In the low-energy limit, the Galileon term will not be significant. However, at high energies $S_{4}$ plays a crucial role in the dynamics. We will discuss the implications of this in Sec. IV.

\section{Coupling to the electromagnetic field}

The action (9) is invariant under the global transformation, i.e., $\pi \rightarrow \pi e^{-i e \theta}$, where $\theta$ is a constant parameter and $e$ is the electric charge. However, the action is not invariant if the parameter $\theta$ is space-time dependent or the local $U(1)$ gauge transformation. In order for the action to be invariant under the transformation, $\pi \rightarrow \pi e^{-i e \theta(x)}$ and $\pi^{*} \rightarrow \pi e^{i e \theta(x)}$, we need to replace the partial derivatives as

$$
\begin{aligned}
\partial_{\mu} \rightarrow D_{\mu} & \equiv \partial_{\mu}+i e A_{\mu} \quad \text { and } \\
\partial_{\mu} \partial^{\nu} \rightarrow D_{\mu} D^{\nu} & =\left(\partial_{\mu}+i e A_{\mu}\right)\left(\partial^{\nu}+i e A^{\nu}\right)
\end{aligned}
$$

where $A_{\mu}$ is the electromagnetic field vector. Under the following gauge transformations 


$$
\pi \rightarrow \pi e^{-i e \theta(x)} ; \quad \pi^{*} \rightarrow \pi e^{i e \theta(x)} ; \quad A_{\mu} \rightarrow A_{\mu}+\partial_{\mu} \theta
$$

the action (9) is invariant. Appendix B contains the details of the calculation. The complete Galileon scalar electrodynamics action in flat space-time is given by

$$
\begin{aligned}
S_{\text {flat }}^{\mathrm{G}}= & \frac{1}{2} \int d^{4} x D_{\mu} \pi D^{\mu} \pi^{*}+\frac{\omega}{2 \lambda_{\pi}^{6}} \int d^{4} x\left[2 D_{\mu} D^{\mu} \pi^{*} D_{\nu} D^{\nu} \pi D_{\alpha} \pi D^{\alpha} \pi^{*}-2 D_{\mu} D_{\nu} \pi^{*} D^{\mu} D^{\nu} \pi D_{\alpha} \pi D^{\alpha} \pi^{*}\right. \\
& \left.+\left(D_{\mu} D^{\mu} \pi D_{\nu} \pi^{*} D^{\nu} D^{\alpha} \pi D_{\alpha} \pi^{*}-D^{\mu} D^{\nu} \pi D_{\nu} D_{\alpha} \pi D_{\mu} \pi^{*} D^{\alpha} \pi^{*}+\text { c.c. }\right)\right]-\frac{1}{4} \int d^{4} x F_{\mu \nu} F^{\mu \nu}
\end{aligned}
$$

where $F_{\mu \nu}=\partial_{\mu} A_{\nu}-\partial_{\nu} A_{\mu}$ is the electromagnetic field tensor. Unlike scalar Galileons, there exists a no-go theorem that states that higher derivative vector Galileons cannot be constructed in flat space-time [19,24]. Thus, for the Galileon scalar electrodynamics in the flat space-time, the nonlinear part of the electromagnetic fields appear through the gauge coupling $D_{\mu}$. It is important to note that the action (13), in addition to U(1) gauge invariance, also satisfies the Galilean symmetry (for $\pi$ and $\pi^{*}$ ), and hence, we refer to this action as Galileon scalar electrodynamics. In Sec. IV, we show that the Galileon term leads to interesting features in inflationary dynamics.

\section{GALILEON SCALAR ELECTRODYNAMICS IN CURVED SPACE-TIME}

One of the critical features of the action of the Galileon field (9) is that it contains second derivatives of the field. Assuming a minimal coupling of the matter and gravity leads to $\partial_{\mu} \rightarrow \nabla_{\mu}$. While the partial derivatives $\partial_{\mu} \partial_{\nu}$ commute, this is not the case for covariant derivatives. We need to take into account the commutation properties of the covariant derivatives. The procedure we will adopt is similar to that of Deffayat et al. [27]. However, due to complex scalar fields, there are some differences in the final expression.

\section{A. Coupling to gravity}

For the minimal coupling, Galilean symmetry is preserved for $\mathcal{L}_{2}$ defined in (4). However, the Galilean symmetry is broken explicitly for the fourth-order Lagrangian (6). Assuming a minimal coupling of the complex field with gravity, the fourth-order action (7) becomes

$$
\begin{aligned}
S_{4}^{\min }= & \frac{\omega}{2 \lambda_{\pi}^{6}} \int d^{4} x \sqrt{-g}\left[2 \square \pi^{*} \square \pi \nabla_{\alpha} \pi \nabla^{\alpha} \pi^{*}\right. \\
& -2 \nabla_{\mu} \nabla_{\nu} \pi^{*} \nabla^{\mu} \nabla^{\nu} \pi \nabla_{\alpha} \pi \nabla^{\alpha} \pi^{*} \\
& +\left(\square \pi \nabla_{\nu} \pi^{*} \nabla^{\nu} \nabla^{\alpha} \pi \nabla_{\alpha} \pi^{*}\right. \\
& \left.\left.-\nabla^{\mu} \nabla^{\nu} \pi \nabla_{\nu} \nabla_{\alpha} \pi \nabla_{\mu} \pi^{*} \nabla^{\alpha} \pi^{*}+\text { c.c. }\right)\right] .
\end{aligned}
$$

Here $\nabla_{\alpha}$ denotes the covariant derivative with respect to the metric $g_{\mu \nu}$ and $\square=\nabla_{\mu} \nabla^{\mu}$. Varying the action (14) with respect to $\pi$ yields the equation of motion of $\pi$. Using the commutation properties of covariant derivatives as given in Appendix $\mathrm{C}$, we obtain the following equation of motion:

$$
\begin{aligned}
\mathcal{E}_{4}^{\min }= & \frac{\omega}{2 \lambda_{\pi}^{6}}\left[-2 \nabla_{\alpha} \pi \nabla^{\alpha} \pi^{*} \nabla^{\nu} \nabla^{\mu} \pi^{*} R_{\mu \nu}-\nabla_{\alpha} \pi \nabla^{\alpha} \pi^{*} \nabla^{\mu} \pi^{*} \nabla_{\mu} R-\nabla^{\mu} \pi^{*} \nabla^{\alpha} \pi^{*} \nabla^{\rho} \pi \nabla_{\rho} R_{\alpha \mu}\right. \\
& -\nabla^{\mu} \pi^{*} \nabla^{\alpha} \pi^{*} \nabla_{\mu} \nabla^{\nu} \pi R_{\nu \alpha}+2 \nabla^{\mu} \pi^{*} \nabla^{\alpha} \pi^{*} \nabla^{\nu} \nabla^{\rho} \pi R_{\rho \mu \alpha \nu}-3 \nabla^{\alpha} \nabla^{\nu} \pi \nabla_{\alpha} \pi^{*} \nabla^{\mu} \pi^{*} R_{\mu \nu} \\
& -2 \nabla^{\mu} \pi^{*} \nabla_{\alpha} \pi \nabla^{\nu} \nabla^{\alpha} \pi^{*} R_{\mu \nu}+\nabla^{\nu} \pi^{*} \nabla^{\alpha} \pi^{*} \square \pi R_{\nu \alpha}-2 \nabla^{\nu} \nabla^{\alpha} \pi^{*} \nabla_{\alpha} \pi^{*} \nabla^{\mu} \pi R_{\mu \nu} \\
& \left.+2 \square \pi^{*} \nabla_{\nu} \nabla_{\alpha} \pi \nabla^{\nu} \nabla^{\alpha} \pi^{*}-\left(\square \pi^{*}\right)^{2} \square \pi-2 \nabla_{\mu} \nabla_{\nu} \pi^{*} \nabla^{\mu} \nabla^{\alpha} \pi^{*} \nabla^{\nu} \nabla_{\alpha} \pi+\square \pi \nabla^{\mu} \nabla^{\nu} \pi^{*} \nabla_{\mu} \nabla_{\nu} \pi^{*}\right]
\end{aligned}
$$

where $R_{\rho \mu \alpha \nu}, R_{\mu \nu}$, and $R$ are Riemann tensor, Ricci tensor, and Ricci scalar respectively. One can immediately notice that the second and third terms in the right-hand side of the above contain third-order derivative terms. Thus, the minimal coupling of the matter with gravity leads to the higher-derivative equations of motion. As mentioned in the Introduction, it is possible to cancel these higherderivative terms adding suitable terms in the action [24,27]. The following nonminimal action

$$
\begin{aligned}
S_{4}^{\mathrm{nm}}= & -\frac{\omega}{4 \lambda_{\pi}^{6}} \int d^{4} x \sqrt{-g} \nabla_{\alpha} \pi \nabla^{\alpha} \pi^{*} \nabla_{\mu} \pi \nabla_{\nu} \pi^{*} g^{\mu \nu} R \\
& -\frac{\omega}{4 \lambda_{\pi}^{6}} \int d^{4} x \sqrt{-g}\left[\nabla_{\alpha} \pi \nabla^{\alpha} \pi \nabla_{\mu} \pi^{*} \nabla_{\nu} \pi^{*}\left(R^{\mu \nu}-\frac{1}{4} g^{\mu \nu} R\right)\right. \\
& + \text { c.c. }]
\end{aligned}
$$

can remove the higher-derivative terms that appear in Eq. (15). See Appendixes C and E for details. If we add 
the above nonminimal action in the action (14) then it will cancel all the higher-order derivative terms in the equation of motion. So varying the action $S_{4}^{\mathrm{min}}+S_{4}^{\mathrm{nm}}$ with respect to $\pi$ gives the equation of motion [see Appendix Eq. (C5)]. Hence, the complete Galileon complex scalar action in an arbitrary curved space-time is given by

$$
S_{\text {Curved }}=S_{2}+S_{4}^{\min }+S_{4}^{\mathrm{nm}} .
$$

This is one of the key results regarding which we would like to stress the following: First, the nonminimal coupling terms in (16) is different for the complex scalar as compared to the nonminimal terms that arises for the real scalar field [27]. Second, as expected, the nonminimal coupling terms vanish and the above action matches with flat space time action (9). Third, in the limit of $\pi=\pi^{*}, S_{4}^{\min }$, and $S_{4}^{\text {nm }}$ reduce to

$$
\begin{aligned}
\left.S_{4}^{\min }\right|_{\pi=\pi^{*}}= & \frac{\omega}{\lambda_{\pi}^{6}} \int d^{4} x \sqrt{-g}\left[(\square \pi)^{2} \nabla_{\alpha} \pi \nabla^{\alpha} \pi+\square \pi \nabla_{\nu} \pi \nabla^{\nu} \nabla^{\alpha} \pi \nabla_{\alpha} \pi\right. \\
& \left.-\nabla_{\mu} \nabla_{\nu} \pi \nabla^{\mu} \nabla^{\nu} \pi \nabla_{\alpha} \pi \nabla^{\alpha} \pi-\nabla^{\mu} \nabla^{\nu} \pi \nabla_{\nu} \nabla_{\alpha} \pi \nabla_{\mu} \pi \nabla^{\alpha} \pi\right], \\
\left.S_{4}^{\mathrm{nm}}\right|_{\pi=\pi^{*}}= & -\frac{\omega}{4 \lambda_{\pi}^{6}} \int d^{4} x \sqrt{-g} \nabla_{\alpha} \pi \nabla^{\alpha} \pi \nabla_{\mu} \pi \nabla_{\nu} \pi g^{\mu \nu} R \\
& -\frac{\omega}{2 \lambda_{\pi}^{6}} \int d^{4} x \sqrt{-g} \nabla_{\alpha} \pi \nabla^{\alpha} \pi \nabla_{\mu} \pi \nabla_{\nu} \pi\left(R^{\mu \nu}-\frac{1}{4} g^{\mu \nu} R\right) .
\end{aligned}
$$

Although the above action looks different compared to that of Deffayet et al. [27], it is possible to show that the two actions are related by a boundary term. In Appendix D, we have explicitly shown that the above action for the real scalar field is identical to the action used in Ref. [27]. Hence, the equations of motion from the above action match with the equations of motion derived in Ref. [27].

\section{B. Coupling to the electromagnetic field}

Like in the flat space-time, the action (17) is invariant under the global transformation, i.e., $\pi \rightarrow \pi e^{-i e \theta}$, where $\theta$ is a constant parameter and $e$ is the electric charge. In order for the action to be invariant under the local gauge transformation, we can replace $\nabla_{\mu} \rightarrow \mathcal{D}_{\mu} \equiv \nabla_{\mu}+i e A_{\mu}$ in action (17). Beside this, the electromagnetic field will have additional Galileon terms that vanish in the flat space-time [24]. The complete Galileon scalar electrodynamics action in curved space time is given by

$$
\begin{aligned}
S_{\text {Curved }}^{\mathrm{G}}= & \frac{1}{2} \int d^{4} x \sqrt{-g} \mathcal{D}_{\mu} \pi \mathcal{D}^{\mu} \pi^{*}+\frac{\omega}{2 \lambda_{\pi}^{6}} \int d^{4} x \sqrt{-g}\left[2 \mathcal{D}_{\mu} \mathcal{D}^{\mu} \pi^{*} \mathcal{D}_{\nu} \mathcal{D}^{\nu} \pi \mathcal{D}_{\alpha} \pi \mathcal{D}^{\alpha} \pi^{*}\right. \\
& \left.-2 \mathcal{D}_{\mu} \mathcal{D}_{\nu} \pi^{*} \mathcal{D}^{\mu} \mathcal{D}^{\nu} \pi \mathcal{D}_{\alpha} \pi \mathcal{D}^{\alpha} \pi^{*}+\left(\mathcal{D}_{\mu} \mathcal{D}^{\mu} \pi \mathcal{D}_{\nu} \pi^{*} \mathcal{D}^{\nu} \mathcal{D}^{\alpha} \pi \mathcal{D}_{\alpha} \pi^{*}-\mathcal{D}^{\mu} \mathcal{D}^{\nu} \pi \mathcal{D}_{\nu} \mathcal{D}_{\alpha} \pi \mathcal{D}_{\mu} \pi^{*} \mathcal{D}^{\alpha} \pi^{*}+\text { c.c. }\right)\right] \\
& \times \frac{\omega}{4 \lambda_{\pi}^{6}} \int d^{4} x \sqrt{-g}\left[\mathcal{D}_{\alpha} \pi \mathcal{D}^{\alpha} \pi \mathcal{D}_{\mu} \pi^{*} \mathcal{D}_{\nu} \pi^{*}\left(R^{\mu \nu}-\frac{1}{4} g^{\mu \nu} R\right)+\text { c.c. }\right] \\
& -\frac{\omega}{4 \lambda_{\pi}^{6}} \int d^{4} x \sqrt{-g} \mathcal{D}_{\alpha} \pi \mathcal{D}^{\alpha} \pi^{*} \mathcal{D}_{\mu} \pi \mathcal{D}_{\nu} \pi^{*} g^{\mu \nu} R-\frac{1}{4} \int d^{4} x F_{\mu \nu} F^{\mu \nu}+S_{\mathrm{VEG}}
\end{aligned}
$$

where the last term $S_{\mathrm{VEG}}$ is the vector Galileon action obtained in Ref. [24]. We have listed the terms in Appendix F. We will use this action to study the effects of the Galileon term in the early Universe.

\section{Fixing the value of $\omega$}

$\lambda_{\pi}$ is the new coupling constant of the model and can only be fixed with observations. As mentioned in Sec. II B $\omega$ can take either +1 or -1 . In this subsection, we fix the value of $\omega$ by evaluating the energy density corresponding to the minimal and nonminimal terms in (19) in the Coulomb gauge $\left(A^{0}=0, \partial_{i} A^{i}=0\right)$.

To make the calculations transparent, we evaluate the energy density in the Friedmann-Robertson-Walker (FRW) background which includes an arbitrary Lapse function $N(t)$ :

$$
d s^{2}=N^{2}(t) d t^{2}-a^{2}(t)\left(d x^{2}+d y^{2}+d z^{2}\right)
$$

where $a(t)$ is the scale factor. To satisfy the homogeneity and isotropy of the FRW background, the Galileon scalar 
electrodynamics must satisfy the condition $A^{i}=0$. The equation of motion of $\pi$ corresponding to $S_{4}^{\mathrm{min}}+S_{4}^{\mathrm{nm}}$ is given by [using Eq. (C5)]:

$$
\begin{aligned}
2 \dot{\pi}^{*} \ddot{\pi}^{*} \dot{\pi}\left(\frac{\dot{a}}{a}\right)^{2}-2\left(\dot{\pi}^{*}\right)^{2} \dot{\pi}\left(\frac{\dot{a}}{a}\right)^{2} \frac{\dot{N}}{N}+33\left(\dot{\pi}^{*}\right)^{2} \dot{\pi}\left(\frac{\dot{a}}{a}\right)^{3} \\
+\left(\dot{\pi}^{*}\right)^{2} \ddot{\pi}\left(\frac{\dot{a}}{a}\right)^{2}-\left(\dot{\pi}^{*}\right)^{2} \dot{\pi}\left(\frac{\dot{a}}{a}\right)^{2} \frac{\dot{N}}{N}+8\left(\dot{\pi}^{*}\right)^{2} \dot{\pi}\left(\frac{\ddot{a}}{a}\right)\left(\frac{\dot{a}}{a}\right) \\
\quad-8\left(\dot{\pi}^{*}\right)^{2} \dot{\pi}\left(\frac{\dot{a}}{a}\right)^{2}\left(\frac{\dot{N}}{N}\right)=0
\end{aligned}
$$

where $H(t)=\dot{a}(t) / a(t)$. Varying the action $S_{4}^{\min }+S_{4}^{\mathrm{nm}}$ with respect to the $g^{00}=N^{-2}(t)$ leads to

$$
\begin{aligned}
\delta S_{4}^{\mathrm{min}} & +\delta S_{4}^{\mathrm{nm}} \\
= & -\frac{3 \omega}{2 \lambda_{\pi}^{6}} \int d^{4} x \frac{a^{3}}{N^{2}}\left[79\left(\dot{\pi}^{*}\right)^{2} \dot{\pi} \ddot{\pi} H(t)+79(\dot{\pi})^{2} \dot{\pi}^{*} \ddot{\pi}^{*} H(t)\right. \\
& \left.+20\left(\dot{\pi}^{*}\right)^{2}(\dot{\pi})^{2} H^{2}(t)+23\left(\dot{\pi}^{*}\right)^{2}(\dot{\pi})^{2}\left(\frac{\ddot{a}}{a}\right)\right] \delta N
\end{aligned}
$$

Using the definition

$$
T_{00}=\frac{2}{\sqrt{-g}} \frac{\delta S}{\delta g^{00}}=-\frac{N^{2}}{a^{3}} \frac{\delta S}{\delta N},
$$

we get

$$
\rho=T_{0}^{0}=\rho=\frac{3 \omega}{2 \lambda_{\pi}^{6}}\left[\frac{79}{2} \partial_{0} N_{1} H+20 N_{1} H^{2}+23 \frac{\ddot{a}}{a} N_{1}\right]
$$

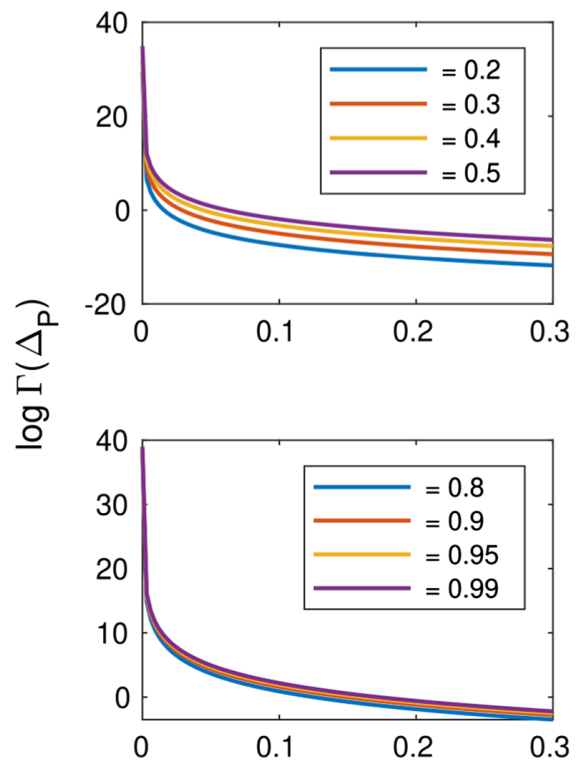

where $N_{1}=|\dot{\pi}|^{4}$. In order to get the value of $\omega$ we simplify the analysis further by setting $N(t)=1$ and taking the limit, $\pi=\pi^{*}$. Thus, the energy density and equation of motion reduce to

$\rho=\frac{3 \omega}{2 \lambda_{\pi}^{6}}\left[2 \times 79(\dot{\pi})^{3} \ddot{\pi} H+20(\dot{\pi})^{4} H^{2}+23(\dot{\pi})^{4}\left(H^{2}+\dot{H}\right)\right]$,

$$
\ddot{\pi}+\frac{41}{3} \dot{\pi} H+\frac{8}{3} \dot{\pi}\left(\frac{\dot{H}}{H}\right)=0 .
$$

Substituting Eq. (26) in Eq. (25), we get

$$
\rho=-\frac{\omega \dot{\pi}^{4} H^{2}}{2 \lambda_{\pi}^{6}}\left[6349+1195 \frac{\dot{H}}{H^{2}}\right] .
$$

Note that $\dot{H} / H^{2}=-\epsilon$ which is a slow-roll parameter. During inflation, when the nonlinear terms contribution cannot be ignored, $\epsilon<1$; hence the quantity in the square bracket is positive. The condition that the energy density is positive implies that $\omega=-1$.

\section{APPLICATIONS TO THE EARLY UNIVERSE}

To extract interesting features of the Galileon model, in this section, we show that the action (19) leads to accelerated expansion in the early Universe. We obtained the energy density of the Galileon field in (25). Using the same procedure, the pressure of the Galileon field is given by
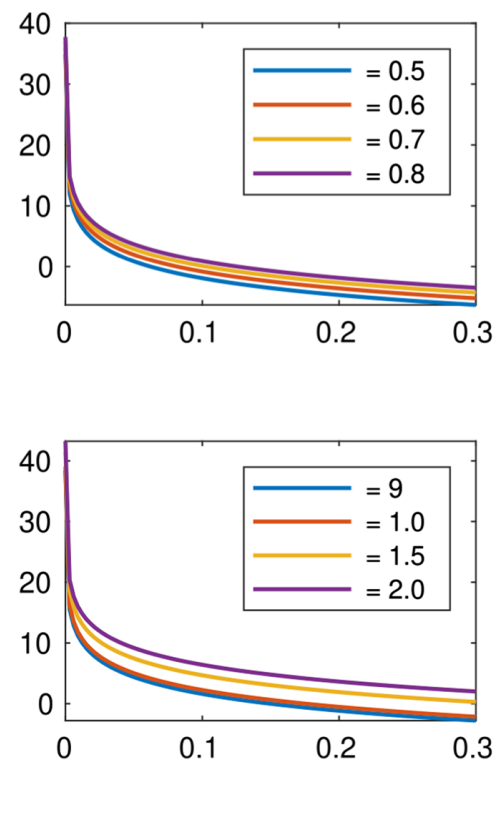

FIG. 1. Plot of $\log \Gamma\left(\Delta_{P}\right)$ versus $\Delta_{P}$ for different values of $\delta_{P}$. Note that for a range of values of the two parameters, $\Gamma$ is also zero. 


$$
p=\frac{\omega}{2 \lambda_{\pi}^{6}}\left[17 \dot{N}_{1} H-72 N_{1} H^{2}+8 \frac{\ddot{a}}{a} N_{1}+3 \ddot{N}_{1}\right] .
$$

Taking the case that the phase variation of the complex field is almost constant, the Friedmann equation becomes

$$
1=\frac{\dot{\pi}^{4}}{6 M_{P}^{2} \lambda_{\pi}^{6}}[6349-1195 \epsilon]
$$

Inverting the above equation, we get

$$
\epsilon=\frac{6349}{1195}[1-\Gamma] \quad \text { where } \Gamma=\frac{6 M_{P}^{2} \lambda_{\pi}^{6}}{6349 \dot{\pi}^{4}} .
$$

In order for $\epsilon$ to be less than unity, $\Gamma \simeq 1$. To identify the parameter space of $\left(\dot{\pi}, \lambda_{\pi}\right)$ that can lead to $\Gamma \simeq 1$, we define $\dot{\pi} \equiv \Delta_{P} M_{P}^{2}, \lambda_{\pi} \equiv \delta_{P} M_{P}$ where $\Delta_{P}$ and $\delta_{P}$ are dimensionless quantities. Figure 1 contains the plot of $\log \Gamma$ as a function of $\Delta_{P}$ for different values of $\delta_{P}$. We notice the following: First, the value of $\Gamma$ is weakly dependent on $\delta_{P}$. In other words, for different values of $\delta_{P}$, the value of $\Gamma$ is almost the same. Second, for large values of $\Delta_{P}, \Gamma$ is almost close to unity and hence, the slow-roll parameter is very small. More specifically, for $\Delta_{P}$ in the range $10^{-5}$ to 0.3 , the slow-roll parameter is of the order of 0.1 . Thus, for a large parameter range, the model can lead to an extended period of inflation.

\section{CONCLUSIONS AND DISCUSSIONS}

In this work, we constructed a Galileon scalar electrodynamics action, which preserves Galilean symmetry and local gauge invariance. Due to the complex scalar field, the number of Galilean symmetry invariant terms are reduced. For an earlier result, in a different context, see Ref. [28]. In the flat space-time, we have explicitly shown that the equations of motion are second order. In curved space-time, due to the noncommutative nature of the covariant derivatives, the minimal coupling of the matter and gravity term leads to higher derivatives in the equation of motion. We introduced nonminimal coupling terms to the Galileon field that makes the equations of motion second order in an arbitrary curved space-time. The nonminimal coupling term is different compared to the one used in the literature for the real scalar field [27]. However, in the real scalar field limit, the complex scalar Galileon action is identical to the real scalar Galileon action obtained by Deffayet et al. [27].

As an application of the model, we considered the case when the Galileon scalar electrodynamics dominated the early Universe. We have shown that for a range of parameters, the model leads to inflation. One possible application of the model is to study the perturbations generated during the inflation dominated by the model. Since the model breaks conformal invariance, this can generate primordial magnetic fields [24]. We hope to report this in more detail shortly.

The model has two free parameters: $\lambda_{\pi}$ and $\lambda_{\mathrm{VG}}$. In the flat FRW background, the electromagnetic field vanishes and hence the scale of inflation fixes $\lambda_{\pi}$. The other parameter $\lambda_{\mathrm{VG}}$ will play a critical role in the first-order perturbations. In principle, the two parameters can be different.

The model presented here can be part of the scalarvector-galileon (SVG) theories. However, as mentioned earlier, not all SVG gravity theories are gauge invariant, while our model is gauge invariant [25]. The complexification of a gauge-invariant subset of the SVG theories should be identical to our model with an appropriate choice of coefficients $A_{i}, B_{i}, C_{i}, D_{i}$ (for $i=1,2$ ) and $D_{3}$. We hope to report this elsewhere.

\section{ACKNOWLEDGMENTS}

The authors wish to thank Debottam Nandi for useful discussions. The authors thank the anonymous referee for raising a point which clarified an important issue in the work. The MHRD fellowship at IIT Bombay financially supports A. K. This work is supported by the ISRORespond grant. Further, we thank Kasper Peeters for his useful program Cadabra [29,30], and useful algebraic calculations with it.

\section{APPENDIX A: FIXING THE COEFFICIENTS OF $\mathcal{L}_{4}$}

In this appendix, we fix all the coefficients in the Lagrangian (5). Demanding that the Lagrangian is invariant under the Galileon transformation (2) leads to the following constraint:

$$
\begin{aligned}
& 2 A_{1} \partial_{\mu} \partial^{\mu} \pi^{*} \partial_{\nu} \partial^{\nu} \pi^{*} \partial_{\alpha} \pi a^{\alpha}+A_{2} \partial_{\mu} \partial^{\mu} \pi^{*} \partial_{\nu} \partial^{\nu} \pi \partial_{\alpha} \pi^{*} a^{\alpha}+B_{1}\left(\partial_{\mu} \partial^{\mu} \pi^{*} \partial^{\nu} \partial^{\alpha} \pi \partial_{\nu} \pi^{*} a_{\alpha}+\partial_{\mu} \partial^{\mu} \pi^{*} \partial^{\nu} \partial^{\alpha} \pi \partial_{\alpha} \pi a_{\nu}{ }^{*}\right) \\
& \quad+2 B_{2} \partial_{\mu} \partial^{\mu} \pi^{*} \partial^{\nu} \partial^{\alpha} \pi^{*} \partial_{\nu} \pi a_{\alpha}+2 C_{1} \partial_{\mu} \partial_{\nu} \pi^{*} \partial^{\mu} \partial^{\nu} \pi^{*} \partial^{\alpha} \pi a_{\alpha}+C_{2} \partial_{\mu} \partial_{\nu} \pi^{*} \partial^{\mu} \partial^{\nu} \pi \partial^{\alpha} \pi^{*} a_{\alpha} \\
& \quad+2 D_{1} \partial^{\mu} \partial^{\nu} \pi^{*} \partial_{\nu} \partial_{\alpha} \pi^{*} \partial_{\mu} \pi a^{\alpha}+D_{2} \partial^{\mu} \partial^{\nu} \pi^{*} \partial_{\nu} \partial_{\alpha} \pi \partial_{\mu} \pi^{*} a^{\alpha}+D_{3} \partial^{\mu} \partial^{\nu} \pi \partial_{\nu} \partial_{\alpha} \pi^{*} \partial_{\mu} \pi^{*} a^{\alpha}+\text { c.c. }=0
\end{aligned}
$$

Here, c.c. denotes the complex conjugate; we have considered only first-order terms in $a^{\mu}$ and have ignored the quadratic terms of the infinitesimal shift parameters. All the first-order terms in Eq. (A1) either do not contribute or contribute total derivative terms. After integrating by parts (and ignoring the total derivative terms as they do not contribute to the action) and collecting similar terms, we get 


$$
\begin{aligned}
& {\left[\left(2 A_{1}-B_{1}\right)\left(\square \pi^{*}\right)^{2} \partial_{\alpha} \pi+\left(A_{2}-2 B_{2}-B_{1}\right) \square \pi^{*} \square \pi \partial_{\alpha} \pi^{*}-\left(B_{1}+2 C_{1}\right) \partial^{\nu} \square \pi^{*} \partial_{\nu} \pi^{*} \partial_{\alpha} \pi\right.} \\
& \quad-\left(B_{1}+D_{3}\right) \partial^{\mu} \square \pi \partial_{\mu} \pi^{*} \partial_{\alpha} \pi^{*}+\left(C_{2}-2 D_{1}-D_{3}\right) \partial_{\mu} \partial_{\nu} \pi^{*} \partial^{\mu} \partial^{\nu} \pi \partial_{\alpha} \pi^{*} \\
& \left.\quad-\left(2 D_{1}+2 B_{2}\right) \partial^{\mu} \square \pi^{*} \partial_{\mu} \pi \partial_{\alpha} \pi^{*}-\left(2 C_{1}-D_{2}\right) \partial_{\mu} \partial_{\nu} \pi^{*} \partial^{\nu} \partial_{\alpha} \pi \partial^{\mu} \pi^{*}\right] a^{\alpha}=0 .
\end{aligned}
$$

The only way to satisfy the above equation for an arbitrary $\pi$ and $\pi^{*}$ is that all the coefficients vanish. This leads to the following relations:

$$
\begin{aligned}
& B_{1}=2 A_{1} ; \quad C_{1}=-A_{1}, \quad B_{2}=-A_{1}+\frac{A_{2}}{2}=-E, \quad C_{2}=-A_{2} ; \\
& D_{2}=-2 A_{1}, \quad D_{1}=A_{1}-\frac{A_{2}}{2}=E, \quad D_{3}=-2 A_{1} .
\end{aligned}
$$

It is straightforward to see from the above expressions that all the parameters can be written in terms of two parameters $A_{1}$ and $A_{2}$. Substituting the coefficients (A3) in Eq. (5) leads to the following generic complex scalar Lagrangian $\mathcal{L}_{4}$ :

$$
\begin{aligned}
\mathcal{L}_{4}= & A_{1}\left[\left((\square \pi)^{2} \partial_{\alpha} \pi^{*} \partial^{\alpha} \pi^{*}+2 \square \pi \partial_{\nu} \pi \partial^{\nu} \partial^{\alpha} \pi^{*} \partial_{\alpha} \pi^{*}-\partial_{\mu} \partial_{\nu} \pi \partial^{\mu} \partial^{\nu} \pi \partial^{\alpha} \pi^{*} \partial_{\alpha} \pi^{*}+\text { c.c. }\right)\right. \\
& \left.-2 \partial_{\mu} \pi^{*} \partial^{\mu} \partial^{\nu} \pi \partial_{\nu} \partial_{\alpha} \pi^{*} \partial^{\alpha} \pi\right]+A_{2}\left[\square \pi^{*} \square \pi \partial_{\alpha} \pi^{*} \partial^{\alpha} \pi-\partial_{\mu} \partial_{\nu} \pi^{*} \partial^{\mu} \partial^{\nu} \pi \partial^{\alpha} \pi^{*} \partial_{\alpha} \pi\right] \\
& +E\left[\partial_{\mu} \pi^{*} \partial^{\mu} \partial^{\nu} \pi \partial_{\nu} \partial_{\alpha} \pi \partial^{\alpha} \pi^{*}-\square \pi \partial_{\nu} \pi^{*} \partial^{\nu} \partial^{\alpha} \pi \partial_{\alpha} \pi^{*}+\text { c.c. }\right] .
\end{aligned}
$$

Now, there are only two free parameters $A_{1}$ and $A_{2}$ in the Lagrangain. The action for the above generic Lagrangian (A4) is given by

$$
S_{4}=\int d^{4} x \mathcal{L}_{4}
$$

We can identify the generic action (A5) as the summation of the following actions:

$$
\begin{aligned}
& S_{4}^{(1)}=A_{1} \int d^{4} x\left(\partial_{\mu} \partial^{\mu} \pi \partial_{\nu} \partial^{\nu} \pi \partial_{\alpha} \pi^{*} \partial^{\alpha} \pi^{*}+\text { c.c. }\right), \\
& S_{4}^{(2)}=A_{2} \int d^{4} x \partial_{\mu} \partial^{\mu} \pi^{*} \partial_{\nu} \partial^{\nu} \pi \partial_{\alpha} \pi^{*} \partial^{\alpha} \pi, \\
& S_{4}^{(3)}=2 A_{1} \int d^{4} x\left(\partial_{\mu} \partial^{\mu} \pi \partial_{\nu} \pi \partial^{\nu} \partial^{\alpha} \pi^{*} \partial_{\alpha} \pi^{*}+\text { c.c. }\right), \\
& S_{4}^{(4)}=-E \int d^{4} x\left(\partial_{\mu} \partial^{\mu} \pi \partial_{\nu} \pi^{*} \partial^{\nu} \partial^{\alpha} \pi \partial_{\alpha} \pi^{*}+\text { c.c. }\right), \\
& S_{4}^{(5)}=-A_{1} \int d^{4} x\left(\partial_{\mu} \partial_{\nu} \pi \partial^{\mu} \partial^{\nu} \pi \partial^{\alpha} \pi^{*} \partial_{\alpha} \pi^{*}+\text { c.c. }\right), \\
& S_{4}^{(6)}=-A_{2} \int d^{4} x \partial_{\mu} \partial_{\nu} \pi^{*} \partial^{\mu} \partial^{\nu} \pi \partial^{\alpha} \pi^{*} \partial_{\alpha} \pi, \\
& S_{4}^{(7)}=E \int d^{4} x\left(\partial_{\mu} \pi \partial^{\mu} \partial^{\nu} \pi^{*} \partial_{\nu} \partial_{\alpha} \pi^{*} \partial^{\alpha} \pi+\text { c.c. }\right), \\
& S_{4}^{(8)}=-2 A_{1} \int d^{4} x \partial_{\mu} \pi^{*} \partial^{\mu} \partial^{\nu} \pi^{*} \partial_{\nu} \partial_{\alpha} \pi \partial^{\alpha} \pi, \\
& S_{4}^{(9)}=-2 A_{1} \int d^{4} x \partial_{\mu} \pi^{*} \partial^{\mu} \partial^{\nu} \pi \partial_{\nu} \partial_{\alpha} \pi^{*} \partial^{\alpha} \pi,
\end{aligned}
$$

where

$$
S_{4}=\sum_{i=1}^{9} S_{4}^{(i)} .
$$

Having obtained the generic fourth-order Galileon action (A6), our next step is to calculate the equations of motion of $\pi$ (for $\pi^{*}$ is straightforward). We define the quantity $\mathcal{E}_{i}$ as the variation of the $S^{(i)}{ }_{C G G}$ with respect to $\pi$ (with respect to $\pi^{*}$ for $\left.\mathcal{E}_{i}{ }^{*}\right)$ as

$$
\mathcal{E}_{4}^{i}=\frac{\delta S_{4}^{(i)}}{\delta \pi} .
$$

Hence, the equation of motion for the generic action (A7) is given by

$$
\mathcal{E}_{4}=\sum_{i=1}^{9} \mathcal{E}_{4}^{i} .
$$

Using Eq. (A8), the equations of motion corresponding to nine actions are

$$
\begin{aligned}
\mathcal{E}_{4}^{(1)}= & 2 A_{1}\left[\partial_{\mu} \partial^{\mu} \square \pi \partial_{\alpha} \pi^{*} \partial^{\alpha} \pi^{*}+4 \partial_{\mu} \square \pi \partial_{\alpha} \pi^{*} \partial^{\mu} \partial^{\alpha} \pi^{*}\right. \\
& +2 \square \pi \partial_{\alpha} \square \pi^{*} \partial^{\alpha} \pi^{*}+2 \square \pi \partial_{\mu} \partial_{\alpha} \pi^{*} \partial^{\mu} \partial^{\alpha} \pi^{*} \\
& \left.-2 \partial^{\alpha} \square \pi^{*} \square \pi^{*} \partial_{\alpha} \pi-\left(\square \pi^{*}\right)^{2} \square \pi\right], \quad(\mathrm{A} 10) \\
\mathcal{E}_{4}^{(2)}= & A_{2}\left[\partial_{\mu} \partial^{\mu} \square \pi^{*} \partial_{\alpha} \pi \partial^{\alpha} \pi^{*}+2 \partial_{\mu} \square \pi^{*} \partial^{\mu} \partial^{\alpha} \pi \partial_{\alpha} \pi^{*}\right. \\
& +2 \partial_{\nu} \square \pi^{*} \partial_{\alpha} \pi \partial^{\nu} \partial^{\alpha} \pi^{*}+\square \pi^{*} \partial_{\alpha} \square \pi \partial^{\alpha} \pi^{*} \\
& +\square \pi^{*} \partial^{\alpha} \square \pi^{*} \partial_{\alpha} \pi+2 \square \pi^{*} \partial_{\nu} \partial_{\alpha} \pi \partial^{\nu} \partial^{\alpha} \pi^{*} \\
& \left.-\partial_{\alpha} \square \pi^{*} \square \pi \partial^{\alpha} \pi^{*}-\square \pi^{*} \partial_{\alpha} \square \pi \partial^{\alpha} \pi^{*}-\left(\square \pi^{*}\right)^{2} \square \pi\right],
\end{aligned}
$$




$$
\begin{aligned}
& \mathcal{E}_{4}^{(3)}=2 A_{1}\left[2 \partial^{\alpha} \partial^{\nu} \square \pi^{*} \partial_{\nu} \pi^{*} \partial_{\alpha} \pi+2 \partial^{\nu} \square \pi^{*} \partial^{\alpha} \pi \partial_{\nu} \partial_{\alpha} \pi^{*}+2 \partial^{\alpha} \square \pi^{*} \square \pi^{*} \partial_{\alpha} \pi+\left(\square \pi^{*}\right)^{2} \square \pi\right. \\
& \left.+2 \partial^{\mu} \partial^{\nu} \partial^{\alpha} \pi^{*} \partial_{\mu} \partial_{\nu} \pi \partial_{\alpha} \pi^{*}+2 \partial^{\mu} \partial^{\nu} \partial^{\alpha} \pi^{*} \partial_{\mu} \partial_{\alpha} \pi^{*} \partial_{\nu} \pi+2 \partial^{\nu} \partial^{\alpha} \pi^{*} \partial_{\mu} \partial_{\nu} \pi \partial^{\mu} \partial_{\alpha} \pi^{*}-\square \pi \partial^{\nu} \partial^{\alpha} \pi^{*} \partial_{\nu} \partial_{\alpha} \pi^{*}\right], \\
& \mathcal{E}_{4}^{(4)}=-E\left[2 \partial^{\nu} \square \pi^{*} \partial_{\nu} \partial_{\alpha} \pi \partial^{\alpha} \pi^{*}+4 \partial^{\mu} \partial^{\nu} \partial^{\alpha} \pi \partial_{\mu} \partial_{\nu} \pi^{*} \partial_{\alpha} \pi^{*}+2 \partial^{\mu} \partial^{\nu} \pi^{*} \partial_{\nu} \partial_{\alpha} \pi \partial_{\mu} \partial^{\alpha} \pi^{*}+2 \partial_{\alpha} \partial_{\nu} \square \pi \partial^{\nu} \pi^{*} \partial^{\alpha} \pi^{*}\right. \\
& +2 \partial^{\nu} \square \pi \partial_{\alpha} \partial_{\nu} \pi^{*} \partial^{\alpha} \pi^{*}+2 \partial^{\nu} \square \pi \square \pi^{*} \partial_{\nu} \pi^{*}+2 \partial^{\alpha} \square \pi^{*} \square \pi \partial_{\alpha} \pi^{*}+\square \pi \partial_{\nu} \partial_{\alpha} \pi^{*} \partial^{\nu} \partial^{\alpha} \pi^{*}+\left(\square \pi^{*}\right)^{2} \square \pi \\
& \left.-\partial_{\nu} \square \pi^{*} \partial^{\nu} \partial^{\alpha} \pi^{*} \partial_{\alpha} \pi-2 \square \pi^{*} \partial^{\alpha} \square \pi^{*} \partial_{\alpha} \pi-2 \square \pi^{*} \partial^{\nu} \partial^{\alpha} \pi^{*} \partial_{\nu} \partial_{\alpha} \pi-\partial_{\alpha} \square \pi^{*} \partial^{\nu} \partial^{\alpha} \pi^{*} \partial_{\nu} \pi\right], \\
& \mathcal{E}_{4}^{(5)}=-2 A_{1}\left[\partial_{\nu} \partial^{\nu} \square \pi \partial_{\alpha} \pi^{*} \partial^{\alpha} \pi^{*}+2 \partial^{\nu} \square \pi \partial^{\alpha} \pi^{*} \partial_{\nu} \partial_{\alpha} \pi^{*}+2 \partial^{\mu} \square \pi \partial^{\alpha} \pi^{*} \partial_{\mu} \partial_{\alpha} \pi^{*}+2 \partial^{\mu} \partial^{\nu} \pi \partial_{\nu} \partial_{\alpha} \pi^{*} \partial_{\mu} \partial^{\alpha} \pi^{*}\right. \\
& \left.+2 \partial^{\mu} \partial^{\nu} \pi \partial^{\alpha} \pi^{*} \partial_{\nu} \partial_{\mu} \partial_{\alpha} \pi^{*}-2 \partial_{\nu} \partial_{\mu} \partial_{\alpha} \pi^{*} \partial^{\mu} \partial^{\nu} \pi^{*} \partial^{\alpha} \pi-\square \pi \partial_{\mu} \partial_{\nu} \pi^{*} \partial^{\mu} \partial^{\nu} \pi^{*}\right], \\
& \mathcal{E}_{4}^{(6)}=-A_{2}\left[\partial_{\nu} \partial^{\nu} \square \pi^{*} \partial_{\alpha} \pi \partial^{\alpha} \pi^{*}+\partial_{\nu} \square \pi^{*} \partial^{\nu} \partial^{\alpha} \pi \partial_{\alpha} \pi^{*}+\partial_{\nu} \square \pi^{*} \partial_{\alpha} \pi \partial^{\nu} \partial^{\alpha} \pi^{*}+\partial_{\mu} \square \pi^{*} \partial^{\mu} \partial^{\alpha} \pi \partial_{\alpha} \pi^{*}\right. \\
& +\partial_{\mu} \partial_{\nu} \pi^{*} \partial^{\mu} \partial^{\alpha} \pi \partial^{\nu} \partial_{\alpha} \pi^{*}+\partial_{\mu} \square \pi^{*} \partial_{\alpha} \pi \partial^{\mu} \partial^{\alpha} \pi^{*}+\partial_{\mu} \partial_{\nu} \pi^{*} \partial^{\nu} \partial^{\alpha} \pi \partial^{\mu} \partial_{\alpha} \pi^{*}+\partial_{\mu} \partial_{\nu} \pi^{*} \partial_{\alpha} \pi \partial^{\mu} \partial^{\nu} \partial^{\alpha} \pi^{*} \\
& \left.-\partial^{\mu} \partial^{\nu} \pi \partial^{\alpha} \pi^{*} \partial_{\alpha} \partial_{\mu} \partial_{\nu} \pi^{*}-\square \pi^{*} \partial_{\mu} \partial_{\nu} \pi^{*} \partial^{\mu} \partial^{\nu} \pi\right], \\
& \mathcal{E}_{4}^{(7)}=E\left[2 \partial^{\mu} \partial^{\alpha} \square \pi \partial_{\mu} \pi^{*} \partial_{\alpha} \pi^{*}+4 \partial^{\mu} \partial^{\nu} \partial^{\alpha} \pi \partial_{\nu} \partial_{\mu} \pi^{*} \partial_{\alpha} \pi^{*}+2 \partial_{\mu} \square \pi \square \pi^{*} \partial^{\mu} \pi^{*}+2 \partial_{\alpha} \square \pi \partial_{\mu} \pi^{*} \partial^{\mu} \partial^{\alpha} \pi^{*}\right. \\
& +2 \square \pi^{*} \partial_{\nu} \partial_{\alpha} \pi \partial^{\nu} \partial^{\alpha} \pi^{*}+2 \partial^{\nu} \square \pi^{*} \partial_{\nu} \partial_{\alpha} \pi \partial^{\alpha} \pi^{*}+2 \partial_{\nu} \partial_{\alpha} \pi \partial^{\nu} \partial^{\mu} \pi^{*} \partial_{\mu} \partial^{\alpha} \pi^{*}+2 \partial_{\nu} \partial_{\alpha} \pi \partial_{\mu} \pi^{*} \partial^{\nu} \partial^{\mu} \partial^{\alpha} \pi^{*} \\
& \left.-2 \partial^{\nu} \square \pi^{*} \partial_{\nu} \partial_{\alpha} \pi^{*} \partial^{\alpha} \pi-2 \partial^{\mu} \partial^{\nu} \pi^{*} \partial_{\mu} \partial_{\nu} \partial_{\alpha} \pi^{*} \partial^{\alpha} \pi-2 \partial^{\mu} \partial^{\nu} \pi^{*} \partial_{\nu} \partial_{\alpha} \pi^{*} \partial_{\mu} \partial^{\alpha} \pi\right], \\
& \mathcal{E}_{4}^{(8)}=-2 A_{1}\left[\partial^{\mu} \partial^{\alpha} \square \pi^{*} \partial_{\mu} \pi^{*} \partial_{\alpha} \pi+\partial^{\mu} \square \pi^{*} \partial_{\alpha} \partial_{\mu} \pi^{*} \partial^{\alpha} \pi+\partial^{\mu} \square \pi^{*} \square \pi \partial_{\mu} \pi^{*}+2 \partial_{\alpha} \partial_{\mu} \partial_{\nu} \pi^{*} \partial^{\nu} \partial^{\mu} \pi^{*} \partial^{\alpha} \pi\right. \\
& +\partial_{\mu} \partial_{\nu} \pi^{*} \partial^{\nu} \partial^{\mu} \pi^{*} \square \pi+\partial^{\alpha} \partial^{\mu} \partial^{\nu} \pi^{*} \partial_{\mu} \pi^{*} \partial_{\nu} \partial_{\alpha} \pi+\partial^{\mu} \partial^{\nu} \pi^{*} \partial_{\mu} \partial_{\alpha} \pi^{*} \partial_{\nu} \partial^{\alpha} \pi+\partial^{\mu} \partial^{\nu} \pi^{*} \partial_{\mu} \pi^{*} \partial_{\nu} \square \pi \\
& \left.-\partial^{\mu} \partial^{\nu} \partial^{\alpha} \pi^{*} \partial_{\nu} \partial_{\alpha} \pi \partial_{\mu} \pi^{*}-\partial^{\mu} \partial^{\nu} \pi^{*} \partial_{\nu} \square \pi \partial_{\mu} \pi^{*}-\partial^{\mu} \partial^{\nu} \pi^{*} \partial_{\nu} \partial_{\alpha} \pi \partial^{\alpha} \partial_{\mu} \pi^{*}\right], \\
& \mathcal{E}_{4}^{(9)}=-2 A_{1}\left[\partial^{\mu} \partial^{\alpha} \square \pi^{*} \partial_{\mu} \pi^{*} \partial_{\alpha} \pi+\partial^{\mu} \partial^{\nu} \partial^{\alpha} \pi^{*} \partial_{\nu} \partial_{\mu} \pi^{*} \partial_{\alpha} \pi+\partial^{\mu} \partial^{\nu} \partial^{\alpha} \pi^{*} \partial_{\mu} \pi^{*} \partial^{\nu} \partial^{\alpha} \pi+\partial_{\alpha} \square \pi^{*} \square \pi^{*} \partial^{\alpha} \pi\right. \\
& \left.+\partial_{\alpha} \partial_{\nu} \pi^{*} \partial^{\nu} \square \pi^{*} \partial^{\alpha} \pi+\partial_{\nu} \partial_{\alpha} \pi^{*} \square \pi^{*} \partial^{\nu} \partial^{\alpha} \pi+\partial_{\alpha} \square \pi^{*} \partial_{\mu} \pi^{*} \partial^{\mu} \partial^{\alpha} \pi-\partial^{\mu} \partial^{\nu} \pi \partial_{\nu} \square \pi^{*} \partial_{\mu} \pi^{*}\right] .
\end{aligned}
$$

Thus, the equation of motion for the generic fourth-order action (A7) is

$$
\mathcal{E}_{4}=\left(A_{1}+\frac{A_{2}}{2}\right)\left[\square \pi \partial_{\mu} \partial_{\nu} \pi^{*} \partial^{\mu} \partial^{\nu} \pi^{*}+2 \square \pi^{*} \partial_{\mu} \partial_{\nu} \pi \partial^{\mu} \partial^{\nu} \pi^{*}-2 \partial_{\mu} \partial_{\nu} \pi^{*} \partial^{\nu} \partial^{\alpha} \pi \partial^{\mu} \partial_{\alpha} \pi^{*}-\left(\square \pi^{*}\right)^{2} \square \pi\right] .
$$

To compare with the earlier results [26], we discuss special cases by taking some specific values of the coefficients $A_{1}$ and $A_{2}$.

\section{Case 1: $A_{1}=0$}

The equation of motion for $\pi$ is given by

$$
\mathcal{E}_{4}^{A_{1}=0}=\frac{A_{2}}{2}\left[\square \pi \partial_{\mu} \partial_{\nu} \pi^{*} \partial^{\mu} \partial^{\nu} \pi^{*}+2 \square \pi^{*} \partial_{\mu} \partial_{\nu} \pi \partial^{\mu} \partial^{\nu} \pi^{*}-2 \partial_{\mu} \partial_{\nu} \pi^{*} \partial^{\nu} \partial^{\alpha} \pi \partial^{\mu} \partial_{\alpha} \pi^{*}-\left(\square \pi^{*}\right)^{2} \square \pi\right]
$$

which in the limit $\pi=\pi^{*}$ gives

$$
\mathcal{E}_{4}^{A_{1}=0}=\frac{A_{2}}{2}\left[3 \square \pi \partial_{\mu} \partial_{\nu} \pi \partial^{\mu} \partial^{\nu} \pi-2 \partial_{\mu} \partial_{\nu} \pi \partial^{\nu} \partial^{\alpha} \pi \partial^{\mu} \partial_{\alpha} \pi-(\square \pi)^{3}\right] .
$$




\section{Case 2: $A_{2}=0$}

The equation of motion for $\pi$ is given by

$$
\begin{aligned}
\mathcal{E}_{4}^{A_{2}=0}= & A_{1}\left[\square \pi \partial_{\mu} \partial_{\nu} \pi^{*} \partial^{\mu} \partial^{\nu} \pi^{*}+2 \square \pi^{*} \partial_{\mu} \partial_{\nu} \pi \partial^{\mu} \partial^{\nu} \pi^{*}\right. \\
& \left.-2 \partial_{\mu} \partial_{\nu} \pi^{*} \partial^{\nu} \partial^{\alpha} \pi \partial^{\mu} \partial_{\alpha} \pi^{*}-\left(\square \pi^{*}\right)^{2} \square \pi\right]
\end{aligned}
$$

which in the limit $\pi=\pi^{*}$ gives

$$
\begin{aligned}
\mathcal{E}_{4}^{A_{2}=0}= & A_{1}\left[3 \square \pi \partial_{\mu} \partial_{\nu} \pi \partial^{\mu} \partial^{\nu} \pi\right. \\
& \left.-2 \partial_{\mu} \partial_{\nu} \pi \partial^{\nu} \partial^{\alpha} \pi \partial^{\mu} \partial_{\alpha} \pi-(\square \pi)^{3}\right] .
\end{aligned}
$$

\section{Case 3: $A_{1}=A_{2}$}

The equation of motion for $\pi$ is given by

$$
\begin{aligned}
\mathcal{E}_{4}^{A_{1} \neq A_{2}}= & A_{1}\left[\frac{3}{2} \square \pi \partial_{\mu} \partial_{\nu} \pi^{*} \partial^{\mu} \partial^{\nu} \pi^{*}+3 \square \pi^{*} \partial_{\mu} \partial_{\nu} \pi \partial^{\mu} \partial^{\nu} \pi^{*}\right. \\
& \left.-3 \partial_{\mu} \partial_{\nu} \pi^{*} \partial^{\nu} \partial^{\alpha} \pi \partial^{\mu} \partial_{\alpha} \pi^{*}-\frac{3}{2}\left(\square \pi^{*}\right)^{2} \square \pi\right] \quad(\mathrm{A} 24)
\end{aligned}
$$

which in the limit $\pi=\pi^{*}$ gives

$$
\begin{aligned}
\mathcal{E}_{4}^{A_{1}=A_{2}}= & \frac{3 A_{1}}{2}\left[3 \square \pi \partial_{\mu} \partial_{\nu} \pi \partial^{\mu} \partial^{\nu} \pi\right. \\
& \left.-2 \partial_{\mu} \partial_{\nu} \pi \partial^{\nu} \partial^{\alpha} \pi \partial^{\mu} \partial_{\alpha} \pi-(\square \pi)^{3}\right] .
\end{aligned}
$$

\section{Case 4: $A_{1} \neq A_{2}$}

Tje equation of motion for $\pi$ is given by

$$
\begin{aligned}
\mathcal{E}_{4}^{A_{1} \neq A_{2}}= & \left(A_{1}+\frac{A_{2}}{2}\right)\left[\square \pi \partial_{\mu} \partial_{\nu} \pi^{*} \partial^{\mu} \partial^{\nu} \pi^{*}\right. \\
& +2 \square \pi^{*} \partial_{\mu} \partial_{\nu} \pi \partial^{\mu} \partial^{\nu} \pi^{*}-2 \partial_{\mu} \partial_{\nu} \pi^{*} \partial^{\nu} \partial^{\alpha} \pi \partial^{\mu} \partial_{\alpha} \pi^{*} \\
& \left.-\left(\square \pi^{*}\right)^{2} \square \pi\right]
\end{aligned}
$$

which in the limit $\pi=\pi^{*}$ gives

$$
\begin{aligned}
\mathcal{E}_{4}^{A_{1} \neq A_{2}}= & \left(A_{1}+\frac{A_{2}}{2}\right)\left[3 \square \pi \partial_{\mu} \partial_{\nu} \pi \partial^{\mu} \partial^{\nu} \pi\right. \\
& \left.-2 \partial_{\mu} \partial_{\nu} \pi \partial^{\nu} \partial^{\alpha} \pi \partial^{\mu} \partial_{\alpha} \pi-(\square \pi)^{3}\right] .
\end{aligned}
$$

From the above cases, it is interesting to see that equations of motion are independent of the values of $A_{1}$ and $A_{2}$ and the equations of motion of all four cases are identical to Ref. [26] in flat space-time. Hence, for simplicity, we set $A_{1}=0$ in the Lagrangian for the fourth-order complex scalar Galileon Lagrangian.

\section{APPENDIX B: GAUGE FIXING}

In this appendix, we show that the Galileon complex scalar action (13) is indeed invariant under the Gauge transformation. Replacing $\partial_{\mu} \rightarrow D_{\mu}=\partial_{\mu}+\alpha A_{\mu}$ (where we fix $\alpha$ at the end of the calculation) implies

$$
\begin{aligned}
\partial_{\mu} \partial^{\nu} \pi \rightarrow D_{\mu} D^{\nu} \pi= & \left(\partial_{\mu}+\alpha A_{\mu}\right)\left(\partial^{\nu}+\alpha A^{\nu}\right) \pi \\
= & \partial_{\mu}{ }^{\nu} \pi+\alpha\left(\partial_{\mu} A^{\nu} \pi+A^{\nu} \partial_{\mu} \pi+A_{\mu} \partial^{\nu} \pi\right) \\
& +\alpha \alpha A_{\mu} A^{\nu} \pi .
\end{aligned}
$$

Under the local U(1) gauge transformation, $\pi \rightarrow \pi e^{-i e \theta(x)}$, the above expression becomes

$$
\begin{aligned}
D_{\mu} D^{\nu}\left(\pi e^{-i e \theta}\right)= & e^{-i e \theta}\left(\partial_{\mu}{ }^{\nu} \pi+\alpha \partial_{\mu} A^{\nu} \pi+A^{\nu} \alpha \partial_{\mu} \pi\right. \\
& +A_{\mu} \alpha \partial^{\nu} \pi+A_{\mu} A^{\nu} \alpha \alpha \pi \\
& -i e\left(\partial^{\nu} \pi \partial_{\mu} \theta+\partial_{\mu} \pi \partial^{\nu} \theta+\partial_{\mu}{ }^{\nu} \theta \pi\right. \\
& \left.+A^{\nu} \alpha \partial_{\mu} \theta \pi+A_{\mu} \alpha \partial^{\nu} \theta \pi\right) \\
& +i e i e \partial_{\mu} \theta \partial^{\nu} \theta \pi .
\end{aligned}
$$

Under the gauge transformation $A_{\mu} \rightarrow A_{\mu}+\partial_{\mu} \theta$, Eq. (B2) becomes

$$
\begin{aligned}
D_{\mu} D^{\nu}\left(\pi e^{-i e \theta}\right) \rightarrow & e^{-i e \theta}\left[\partial_{\mu}{ }^{\nu} \pi+\alpha\left(\partial_{\mu} A^{\nu} \pi+\partial_{\mu} \partial^{\nu} \theta \pi+A^{\nu} \partial_{\mu} \pi+\partial^{\nu} \theta \partial_{\mu} \pi+A_{\mu} \partial^{\nu} \pi+\partial_{\mu} \theta \partial^{\nu} \pi\right.\right. \\
& \left.+\alpha A_{\mu} A^{\nu} \pi+\alpha A_{\mu} \partial^{\nu} \theta \pi+\alpha A^{\nu} \partial_{\mu} \theta \pi+\alpha \partial_{\mu} \theta \partial^{\nu} \theta \pi\right)-i e\left(\partial^{\nu} \pi \partial_{\mu} \theta+\partial_{\mu} \pi \partial^{\nu} \theta+\partial_{\mu}{ }^{\nu} \theta \pi\right. \\
& \left.\left.+\alpha A^{\nu} \partial_{\mu} \theta \pi+\alpha \partial^{\nu} \theta \partial_{\mu} \theta \pi+\alpha A_{\mu} \partial^{\nu} \theta \pi+\alpha \partial_{\mu} \partial^{\nu} \theta \pi\right)+i e i e \partial_{\mu} \theta \partial^{\nu} \theta \pi\right] .
\end{aligned}
$$

For $D_{\mu} D^{\nu} \pi$ to be invariant under the local gauge transformations, we set $\alpha=i e$ in Eq. (B3). Hence, the action (9) is invariant under the following simultaneous transformations:

$$
A_{\mu} \rightarrow A_{\mu}+\partial_{\mu} \theta ; \quad \pi \rightarrow \pi e^{-i e \theta(x)}
$$

and the quantity $D_{\mu} D^{\nu} \pi$ will transform as

$$
\begin{aligned}
D_{\mu} D^{\nu}\left(\pi e^{-i e \theta}\right)= & \left(\partial_{\mu}{ }^{\nu} \pi+\alpha \partial_{\mu} A^{\nu} \pi+A^{\nu} \alpha \partial_{\mu} \pi\right. \\
& \left.+A_{\mu} \alpha \partial^{\nu} \pi+A_{\mu} A^{\nu} \alpha \alpha \pi\right) e^{-i e \theta}
\end{aligned}
$$

Similar analysis can be done for $\pi^{*} \rightarrow \pi^{*} e^{i e \theta(x)}$ also. 


\section{APPENDIX C: GALILEON SCALAR ELECTRODYNAMICS IN CURVED SPACE-TIME}

In this appendix, we show that the action (14) leads to higher derivative equations of motion. We also show addition of the nonminimal term leads to second-order equations of motion. The variation of the action (14) with respect to $\pi$ yields the equation of motion:

$$
\begin{aligned}
\mathcal{E}_{4}^{\min }= & \frac{\omega}{2 \lambda_{\pi}^{6}}\left[2 \nabla_{\mu} \nabla^{\mu} \pi^{*} \nabla_{\nu} \nabla_{\alpha} \pi \nabla^{\nu} \nabla^{\alpha} \pi^{*}-\nabla_{\mu} \nabla^{\mu} \pi^{*} \nabla_{\nu} \nabla^{\nu} \pi^{*} \nabla_{\alpha} \nabla^{\alpha} \pi-2 \nabla_{\mu} \nabla_{\nu} \pi^{*} \nabla^{\mu} \nabla^{\alpha} \pi^{*} \nabla^{\nu} \nabla_{\alpha} \pi\right. \\
& +\nabla_{\mu} \nabla^{\mu} \pi \nabla^{\mu} \nabla^{\nu} \pi^{*} \nabla_{\mu} \nabla_{\nu} \pi^{*}+2 \nabla_{\alpha} \pi \nabla^{\alpha} \pi^{*}\left(\nabla^{\nu} \nabla_{\nu} \nabla_{\mu} \nabla^{\mu} \pi^{*}-\nabla^{\nu} \nabla^{\mu} \nabla_{\mu} \nabla_{\nu} \pi^{*}\right) \\
& +\nabla^{\alpha} \pi^{*} \nabla_{\nu} \pi^{*}\left(\nabla^{\mu} \nabla_{\mu} \nabla^{\nu} \nabla_{\alpha} \pi+\nabla^{\alpha} \nabla^{\nu} \nabla_{\mu} \nabla^{\mu} \pi\right)-\nabla^{\alpha} \pi^{*} \nabla_{\mu} \pi^{*}\left(\nabla^{\nu} \nabla^{\mu} \nabla_{\nu} \nabla_{\alpha} \pi+\nabla_{\alpha} \nabla_{\nu} \nabla^{\mu} \nabla^{\nu} \pi\right) \\
& +3 \nabla^{\nu} \nabla^{\alpha} \pi \nabla_{\alpha} \pi^{*}\left(\nabla_{\nu} \nabla_{\mu} \nabla^{\mu} \pi^{*}-\nabla_{\mu} \nabla^{\mu} \nabla_{\nu} \pi^{*}\right)+2 \nabla^{\nu} \nabla^{\alpha} \pi^{*} \nabla_{\alpha} \pi\left(\nabla_{\nu} \nabla_{\mu} \nabla^{\mu} \pi^{*}-\nabla_{\mu} \nabla^{\mu} \nabla_{\nu} \pi^{*}\right) \\
& +\nabla^{\mu} \nabla_{\mu} \pi \nabla^{\alpha} \pi^{*}\left(\nabla_{\nu} \nabla^{\nu} \nabla_{\alpha} \pi^{*}-\nabla_{\alpha} \nabla^{\nu} \nabla_{\nu} \pi^{*}\right)+2 \nabla^{\mu} \nabla^{\nu} \pi^{*} \nabla^{\alpha} \pi^{*}\left(\nabla_{\mu} \nabla_{\nu} \nabla_{\alpha} \pi-\nabla_{\nu} \nabla_{\mu} \nabla_{\alpha} \pi\right) \\
& +\nabla^{\nu} \nabla^{\alpha} \pi^{*} \nabla_{\alpha} \pi^{*}\left(\nabla_{\nu} \nabla_{\mu} \nabla^{\mu} \pi-\nabla_{\mu} \nabla^{\mu} \nabla_{\nu} \pi\right)+2 \nabla^{\mu} \nabla^{\nu} \pi \nabla^{\alpha} \pi^{*}\left(\nabla_{\alpha} \nabla_{\mu} \nabla_{\nu} \pi^{*}-\nabla_{\nu} \nabla_{\alpha} \nabla_{\mu} \pi^{*}\right) \\
& \left.+2 \nabla^{\mu} \nabla^{\nu} \pi^{*} \nabla^{\alpha} \pi\left(\nabla_{\mu} \nabla_{\alpha} \nabla_{\nu} \pi^{*}-\nabla_{\nu} \nabla_{\mu} \nabla_{\alpha} \pi^{*}\right)\right] .
\end{aligned}
$$

Using the following commutation properties of covariant derivatives:

$$
\left[\nabla_{\mu}, \nabla_{\nu}\right] \nabla^{\alpha} \pi=R_{\rho \mu \nu}^{\alpha} \nabla^{\rho} \pi ; \quad \nabla_{\nu} \nabla_{\mu} \nabla_{\alpha} \pi-\nabla_{\alpha} \nabla_{\mu} \nabla_{\nu} \pi=R_{\mu \alpha \nu}^{\rho} \nabla_{\rho} \pi ; \quad \nabla_{\alpha} \nabla_{\nu} \nabla^{\nu} \pi-\nabla_{\nu} \nabla^{\nu} \nabla_{\alpha} \pi=-R_{\nu \alpha} \nabla^{\nu} \pi
$$

we get

$$
\begin{aligned}
\mathcal{E}_{4}^{\min }= & \frac{\omega}{2 \lambda_{\pi}^{6}}\left[-2 \nabla_{\alpha} \pi \nabla^{\alpha} \pi^{*} \nabla^{\nu} \nabla^{\mu} \pi^{*} R_{\mu \nu}-\nabla_{\alpha} \pi \nabla^{\alpha} \pi^{*} \nabla^{\mu} \pi^{*} \nabla_{\mu} R+2 \nabla^{\mu} \pi^{*} \nabla^{\alpha} \pi^{*} \nabla^{\nu} \nabla^{\rho} \pi R_{\rho \mu \alpha \nu}\right. \\
& -\nabla^{\mu} \pi^{*} \nabla^{\alpha} \pi^{*} \nabla_{\mu} \nabla^{\nu} \pi R_{\nu \alpha}-\nabla^{\mu} \pi^{*} \nabla^{\alpha} \pi^{*} \nabla^{\rho} \pi \nabla_{\rho} R_{\alpha \mu}-3 \nabla^{\alpha} \nabla^{\nu} \pi \nabla_{\alpha} \pi^{*} \nabla^{\mu} \pi^{*} R_{\mu \nu} \\
& -2 \nabla^{\mu} \pi^{*} \nabla_{\alpha} \pi \nabla^{\nu} \nabla^{\alpha} \pi^{*} R_{\mu \nu}+\nabla^{\nu} \pi^{*} \nabla^{\alpha} \pi^{*} \nabla^{\mu} \nabla_{\mu} \pi R_{\nu \alpha}-2 \nabla^{\nu} \nabla^{\alpha} \pi^{*} \nabla_{\alpha} \pi^{*} \nabla^{\mu} \pi R_{\mu \nu} \\
& +2 \nabla_{\mu} \nabla^{\mu} \pi^{*} \nabla_{\nu} \nabla_{\alpha} \pi \nabla^{\nu} \nabla^{\alpha} \pi^{*}-\nabla_{\mu} \nabla^{\mu} \pi^{*} \nabla_{\nu} \nabla^{\nu} \pi^{*} \nabla_{\alpha} \nabla^{\alpha} \pi-2 \nabla_{\mu} \nabla_{\nu} \pi^{*} \nabla^{\mu} \nabla^{\alpha} \pi^{*} \nabla^{\nu} \nabla_{\alpha} \pi \\
& +\nabla_{\mu} \nabla^{\mu} \pi \nabla^{\mu} \nabla^{\nu} \pi^{*} \nabla_{\mu} \nabla_{\nu} \pi^{*} .
\end{aligned}
$$

Note that the second and fifth terms in the above equation are higher derivative (of metric) terms. In order to remove these terms we need to add some nonminimal terms in the action in such a way that on varying the total action, all the derivatives of the Ricci tensor and Ricci scalar vanish. The equation of motion for the nonminimal action (16) is given by

$$
\begin{aligned}
\mathcal{E}_{4}^{\mathrm{nm}}= & \frac{\omega}{4 \lambda_{\pi}^{6}}\left[2 \nabla_{\mu} \nabla^{\mu} \pi^{*} \nabla_{\nu} \pi^{*} \nabla^{\nu} \pi R+2 \nabla_{\mu} \nabla_{\nu} \pi \nabla^{\mu} \pi^{*} \nabla^{\nu} \pi^{*} R+2 \nabla_{\mu} \pi^{*} \nabla^{\mu} \pi \nabla^{\alpha} \pi^{*} \nabla_{\alpha} R\right. \\
& -\nabla_{\mu} \nabla^{\mu} \pi \nabla_{\nu} \pi^{*} \nabla^{\nu} \pi^{*} R+2 \nabla_{\mu} \nabla^{\mu} \pi \nabla^{\nu} \pi^{*} \nabla^{\alpha} \pi^{*} R_{\nu \alpha}+4 \nabla^{\alpha} \nabla^{\mu} \pi^{*} \nabla_{\alpha} \pi \nabla^{\nu} \pi^{*} R_{\mu \nu} \\
& \left.+2 \nabla^{\alpha} \pi \nabla^{\mu} \pi^{*} \nabla^{\nu} \pi^{*} \nabla_{\alpha} R_{\mu \nu}+4 \nabla^{\mu} \nabla_{\alpha} \pi^{*} \nabla^{\alpha} \pi^{*} \nabla^{\nu} \pi R_{\mu \nu}+2 \nabla_{\alpha} \pi^{*} \nabla^{\alpha} \pi^{*} \nabla^{\mu} \nabla^{\nu} \pi R_{\mu \nu}\right]
\end{aligned}
$$

If we add the above nonminimal action in the action (14) then it will cancel all the higher-order derivative terms in the equation of motion. So varying the action $S_{4}^{\mathrm{min}}+S_{4}^{\mathrm{nm}}$ with respect to $\pi$, we get

$$
\begin{aligned}
\mathcal{E}^{\prime}= & \frac{\omega}{2 \lambda_{\pi}^{6}}\left[2 \square \pi^{*} \nabla_{\nu} \nabla_{\alpha} \pi \nabla^{\nu} \nabla^{\alpha} \pi^{*}-\left(\square \pi^{*}\right)^{2} \square \pi+\square \pi \nabla^{\mu} \nabla^{\nu} \pi^{*} \nabla_{\mu} \nabla_{\nu} \pi^{*}-2 \nabla_{\mu} \nabla_{\nu} \pi^{*} \nabla^{\mu} \nabla^{\alpha} \pi^{*} \nabla^{\nu} \nabla_{\alpha} \pi\right. \\
& +\square \pi^{*} \nabla_{\mu} \pi^{*} \nabla^{\mu} \pi R-\frac{1}{2} \square \pi \nabla_{\alpha} \pi^{*} \nabla^{\alpha} \pi^{*} R+\nabla_{\alpha} \pi^{*} \nabla_{\mu} \pi^{*} \nabla^{\mu} \nabla^{\alpha} \pi R+2 \square \pi \nabla^{\nu} \pi^{*} \nabla^{\mu} \pi^{*} R_{\nu \mu} \\
& +\nabla_{\alpha} \pi^{*} \nabla^{\alpha} \pi^{*} \nabla^{\nu} \nabla^{\mu} \pi R_{\mu \nu}-2 \nabla_{\alpha} \pi \nabla^{\alpha} \pi^{*} \nabla^{\nu} \nabla^{\mu} \pi^{*} R_{\mu \nu}-\nabla^{\mu} \pi^{*} \nabla^{\alpha} \pi^{*} \nabla_{\mu} \nabla^{\nu} \pi R_{\nu \alpha} \\
& \left.+2 \nabla^{\mu} \pi^{*} \nabla^{\alpha} \pi^{*} \nabla^{\nu} \nabla^{\rho} \pi R_{\rho \mu \alpha \nu}-3 \nabla^{\alpha} \nabla^{\nu} \pi \nabla_{\alpha} \pi^{*} \nabla^{\mu} \pi^{*} R_{\mu \nu}\right] .
\end{aligned}
$$




\section{APPENDIX D: CONSISTENCY CHECK WITH REAL SCALAR GALILEON}

In this section, we show systematically that our fourth-order action $\left(S_{4}^{\min }+S_{4}^{\mathrm{nm}}\right)$ exactly matches with the results of Deffayet et al. [27] in the $\pi=\pi^{*}$ limit. Equation (18) can be written as

$$
\begin{aligned}
S_{4}^{\min }+\left.S_{4}^{\mathrm{nm}}\right|_{\pi=\pi^{*}}= & A_{2} \int d^{4} x \sqrt{-g}\left[(\square \pi)^{2} \nabla_{\alpha} \pi \nabla^{\alpha} \pi+\square \pi \nabla_{\nu} \pi \nabla^{\nu} \nabla^{\alpha} \pi \nabla_{\alpha} \pi-\nabla_{\mu} \nabla_{\nu} \pi \nabla^{\mu} \nabla^{\nu} \pi \nabla_{\alpha} \pi \nabla^{\alpha} \pi-\nabla^{\mu} \nabla^{\nu} \pi \nabla_{\nu} \nabla_{\alpha} \pi \nabla_{\mu} \pi \nabla^{\alpha} \pi\right] \\
& +\frac{A_{2}}{4} \int d^{4} x \sqrt{-g} \nabla_{\alpha} \pi \nabla^{\alpha} \pi \nabla^{\mu} \pi \nabla^{\nu} \pi G_{\mu \nu}-\frac{3 A_{2}}{4} \int d^{4} x \sqrt{-g} \nabla_{\alpha} \pi \nabla^{\alpha} \pi \nabla^{\mu} \pi \nabla^{\nu} \pi R_{\mu \nu}
\end{aligned}
$$

where $G_{\mu \nu}=R_{\mu \nu}-\frac{1}{2} g_{\mu \nu} R$. Concentrating on the last term in the above equation (D1) and using the relation $\left(\nabla_{\mu} \nabla_{\nu} \nabla^{\nu} \pi-\nabla_{\nu} \nabla^{\nu} \nabla_{\mu} \pi\right) \nabla^{\mu} \pi=R_{\mu \nu} \nabla^{\mu} \nabla^{\nu} \pi$, ignoring the total derivative terms, we get

$$
\begin{aligned}
-\frac{3 A_{2}}{4} \nabla_{\alpha} \pi \nabla^{\alpha} \pi \nabla^{\mu} \pi \nabla^{\nu} \pi R_{\mu \nu}= & -\frac{3 A_{2}}{4} \nabla_{\alpha} \pi \nabla^{\alpha} \pi \nabla_{\mu} \nabla^{\mu} \nabla_{\nu} \pi \nabla^{\nu} \pi+\frac{3 A_{2}}{4} \nabla_{\alpha} \pi \nabla^{\alpha} \pi \nabla_{\nu} \nabla^{\mu} \nabla_{\mu} \pi \nabla^{\nu} \pi \\
= & \frac{3 A_{2}}{2} \nabla_{\mu} \nabla_{\alpha} \pi \nabla^{\mu} \nabla^{\nu} \pi \nabla_{\nu} \pi \nabla^{\alpha} \pi+\frac{3 A_{2}}{4} \nabla_{\mu} \nabla_{\nu} \pi \nabla^{\mu} \nabla^{\mu} \pi \nabla^{\nu} \pi \nabla_{\alpha} \pi \nabla^{\alpha} \pi \\
& -\frac{3 A_{2}}{2}(\square \pi)^{2} \nabla_{\alpha} \pi \nabla^{\alpha} \pi-\frac{3 A_{2}}{4} \square \pi \nabla^{\mu} \nabla^{\nu} \pi \nabla_{\mu} \pi \nabla_{\nu} \pi .
\end{aligned}
$$

Using Eqs. (D1) and (D2), we get

$$
\begin{aligned}
S_{4}^{\min }+\left.S_{4}^{\mathrm{nm}}\right|_{\pi=\pi^{*}}= & \frac{A_{2}}{4} \int d^{4} x \sqrt{-g}\left[(\square \pi)^{2} \nabla_{\alpha} \pi \nabla^{\alpha} \pi+\square \pi \nabla_{\nu} \pi \nabla^{\nu} \nabla^{\alpha} \pi \nabla_{\alpha} \pi-\nabla_{\mu} \nabla_{\nu} \pi \nabla^{\mu} \nabla^{\nu} \pi \nabla_{\alpha} \pi \nabla^{\alpha} \pi-\nabla^{\mu} \nabla^{\nu} \pi \nabla_{\nu} \nabla_{\alpha} \pi \nabla_{\mu} \pi \nabla^{\alpha} \pi\right] \\
& +\frac{A_{2}}{4} \int d^{4} x \sqrt{-g} \nabla_{\alpha} \pi \nabla^{\alpha} \pi \nabla^{\mu} \pi \nabla^{\nu} \pi G_{\mu \nu} .
\end{aligned}
$$

Thus, the above action (D3) matches with the action in Ref. [27]. It is important to note that there is an overall sign difference between our result and the one obtained by Deffayet et al. [27]. This is due to the signature convention of the metric and hence our definition of energy-momentum tensor i.e., $T_{\mu \nu}=\frac{2}{\sqrt{-g}} \frac{\delta S}{\delta g^{\mu \nu}}$, differs by an overall negative sing compared to Deffayet et al. [27].

\section{APPENDIX E: GALILEON SCALAR ELECTRODYNAMICS IN CURVED SPACE TIME FOR $\boldsymbol{A}_{2}=0$}

Before discussing the other special case $A_{2}=0$, we obtain the nonminimal coupling terms for the full action. Consider the action corresponding to the full Lagrangian (A4) and varying the action with respect to $\pi$ gives the equation of motion for the generic action in curved spacetime. As our main focus is to obtain the nonminimal action, we collect only the fourthorder derivative terms in the field as we have done in Appendix C. After collecting the fourth-order terms we get

$$
\begin{aligned}
\mathcal{E}_{4}^{\min }= & \frac{A_{2}}{2}\left[2 \nabla_{\alpha} \pi \nabla^{\alpha} \pi^{*}\left(\nabla^{\nu} \nabla_{\nu} \nabla_{\mu} \nabla^{\mu} \pi^{*}-\nabla^{\nu} \nabla^{\mu} \nabla_{\mu} \nabla_{\nu} \pi^{*}\right)\right. \\
& \left.+\nabla_{\alpha} \pi^{*} \nabla^{\mu} \pi^{*}\left(\nabla_{\nu} \nabla^{\nu} \nabla^{\alpha} \nabla_{\mu} \pi+\nabla_{\mu} \nabla^{\alpha} \nabla_{\nu} \nabla^{\nu} \pi-\nabla_{\nu} \nabla^{\alpha} \nabla^{\nu} \nabla_{\mu} \pi-\nabla_{\mu} \nabla_{\nu} \nabla^{\alpha} \nabla^{\nu} \pi\right)\right] \\
& +A_{1}\left[2 \nabla_{\alpha} \pi^{*} \nabla^{\alpha} \pi^{*}\left(\nabla^{\nu} \nabla_{\nu} \nabla_{\mu} \nabla^{\mu} \pi-\nabla^{\nu} \nabla^{\mu} \nabla_{\mu} \nabla_{\nu} \pi\right)\right. \\
& +\nabla^{\alpha} \pi^{*} \nabla^{\mu} \pi^{*}\left(\nabla_{\nu} \nabla^{\alpha} \nabla^{\nu} \nabla_{\mu} \pi+\nabla_{\mu} \nabla_{\nu} \nabla^{\alpha} \nabla^{\nu} \pi-\nabla^{\nu} \nabla_{\nu} \nabla^{\alpha} \nabla_{\mu} \pi-\nabla_{\mu} \nabla^{\alpha} \nabla_{\nu} \nabla^{\nu} \pi\right) \\
& \left.+2 \nabla_{\alpha} \pi^{*} \nabla_{\nu} \pi\left(\nabla^{\mu} \nabla_{\mu} \nabla^{\nu} \nabla^{\alpha} \pi^{*}+\nabla^{\nu} \nabla^{\alpha} \nabla^{\mu} \nabla_{\mu} \pi^{*}-\nabla^{\nu} \nabla^{\mu} \nabla^{\alpha} \nabla_{\mu} \pi^{*}-\nabla_{\mu} \nabla_{\alpha} \nabla^{\mu} \nabla_{\nu} \pi^{*}\right)\right]
\end{aligned}
$$

using the commutation properties of the covariant derivatives and using $(\mathrm{C} 2)$ we get

$$
\begin{aligned}
\mathcal{E}_{4}^{\min }= & -\frac{A_{2}}{2} \nabla_{\alpha} \pi^{*} \nabla^{\alpha} \pi \nabla^{\mu} \pi^{*} \nabla_{\mu} R-A_{1} \nabla_{\alpha} \pi^{*} \nabla^{\alpha} \pi^{*} \nabla^{\mu} \pi \nabla_{\mu} R-\frac{A_{2}}{2} \nabla^{\alpha} \pi^{*} \nabla^{\mu} \pi^{*} \nabla^{\nu} \pi \nabla_{\nu} R_{\alpha \mu} \\
& +A_{1} \nabla^{\alpha} \pi^{*} \nabla^{\mu} \pi^{*} \nabla^{\nu} \pi \nabla_{\nu} R_{\alpha \mu}-2 A_{1} \nabla^{\alpha} \pi^{*} \nabla^{\mu} \pi^{*} \nabla^{\nu} \pi \nabla_{\mu} R_{\alpha \nu} .
\end{aligned}
$$

We now consider the following general nonminimal action 


$$
S_{4}^{\mathrm{nm}}=\int d^{4} x \sqrt{-g} \mathcal{L}_{4}^{\mathrm{nm}}
$$

where

$$
\begin{aligned}
\mathcal{L}_{4}^{\mathrm{nm}}= & -\left[\nabla_{\alpha} \pi^{*} \nabla^{\alpha} \pi^{*} \nabla^{\mu} \pi \nabla^{\nu} \pi+\nabla_{\alpha} \pi \nabla^{\alpha} \pi \nabla^{\mu} \pi^{*} \nabla^{\nu} \pi^{*}\right] \\
& \times\left(g_{1} R_{\mu \nu}+g_{2} g_{\mu \nu} R\right) \\
& -\nabla_{\alpha} \pi^{*} \nabla^{\alpha} \pi \nabla^{\mu} \pi^{*} \nabla^{\nu} \pi\left(h_{1} R_{\mu \nu}+h_{2} g_{\mu \nu} R\right)
\end{aligned}
$$

where $g_{1}, g_{2}, h_{1}$ and $h_{2}$ are arbitrary constants which we will fix later. Variation of the action (E3) with respect to $\pi$ and collecting only derivatives of the Ricci tensor and Ricci scalar terms because we are interested in fixing the coefficients $g_{1}, g_{2}, h_{1}, h_{2}$, we obtain

$$
\begin{aligned}
\mathcal{E}_{4}^{\mathrm{nm}}= & g_{1} \nabla_{\alpha} \pi^{*} \nabla^{\alpha} \pi^{*} \nabla^{\mu} \pi \nabla_{\mu} R+4 g_{2} \nabla_{\alpha} \pi^{*} \nabla^{\alpha} \pi^{*} \nabla^{\mu} \pi \nabla_{\mu} R \\
& +\frac{h_{1}}{2} \nabla_{\alpha} \pi^{*} \nabla^{\alpha} \pi \nabla^{\mu} \pi^{*} \nabla_{\mu} R \\
& +2 h_{2} \nabla_{\alpha} \pi^{*} \nabla^{\alpha} \pi \nabla^{\mu} \pi^{*} \nabla_{\mu} R \\
& +2 g_{1} \nabla^{\mu} \pi^{*} \nabla^{\nu} \pi^{*} \nabla^{\alpha} \pi \nabla_{\alpha} R_{\mu \nu} \\
& +h_{1} \nabla^{\mu} \pi^{*} \nabla^{\nu} \pi \nabla^{\alpha} \pi^{*} \nabla_{\alpha} R_{\mu \nu} .
\end{aligned}
$$

Adding Eqs. (E2) and (E5) such that all the derivative terms of the Ricci tensor and scalar vanish, we obtain the following relations:

$$
\begin{aligned}
-A_{1}+g_{1}+4 g_{2} & =0, \\
-\frac{A_{2}}{2}+A_{1}+2 g_{1} & =0, \\
-\frac{A_{2}}{2}+2 h_{2}+\frac{h_{1}}{2} & =0 \\
-2 A_{1}+h_{1} & =0,
\end{aligned}
$$

which implies

$$
\begin{aligned}
& g_{1}=\frac{A_{2}}{4}-\frac{A_{1}}{2}, \\
& g_{2}=\frac{3 A_{1}}{8}-\frac{A_{2}}{16}, \\
& h_{1}=2 A_{1} \\
& h_{2}=-\frac{A_{1}}{2}+\frac{A_{2}}{4} .
\end{aligned}
$$

Substituting the above values in Eq. (E4), we get

$$
\begin{aligned}
\mathcal{L}_{4}^{\mathrm{nm}}= & \frac{A_{1}}{2}\left[\nabla_{\alpha} \pi^{*} \nabla^{\alpha} \pi^{*} \nabla^{\mu} \pi \nabla^{\nu} \pi+\nabla_{\alpha} \pi \nabla^{\alpha} \pi \nabla^{\mu} \pi^{*} \nabla^{\nu} \pi^{*}\right]\left(R_{\mu \nu}-\frac{3}{4} g_{\mu \nu} R\right) \\
& -\frac{A_{2}}{4}\left[\nabla_{\alpha} \pi^{*} \nabla^{\alpha} \pi^{*} \nabla^{\mu} \pi \nabla^{\nu} \pi+\nabla_{\alpha} \pi \nabla^{\alpha} \pi \nabla^{\mu} \pi^{*} \nabla^{\nu} \pi^{*}\right]\left(R_{\mu \nu}-\frac{1}{4} g_{\mu \nu} R\right) \\
& -2 A_{1} \nabla_{\alpha} \pi^{*} \nabla^{\alpha} \pi \nabla^{\mu} \pi^{*} \nabla^{\nu} \pi\left(R_{\mu \nu}-\frac{1}{4} g_{\mu \nu} R\right)-\frac{A_{2}}{4} \nabla_{\alpha} \pi^{*} \nabla^{\alpha} \pi \nabla^{\mu} \pi^{*} \nabla^{\nu} \pi g_{\mu \nu} R .
\end{aligned}
$$

We note that the above nonminimal action is identical with the $A_{1}=0$ case considered in Appendix C. In the $\pi=\pi^{*}$ limit, this case also leads to identical equations of motion as in Ref. [27].

\section{APPENDIX F: VECTOR GALILEON ACTION $S_{\text {VEG }}$}

In Ref. [24], the authors obtained a vector Galileon model that leads to second-order equations. For completeness, in this Appendix, we have listed below the vector Galileon action. The complete vector Galileon action can be written as

$$
S_{\mathrm{VEC}}=S_{\mathrm{VG}}+\lambda_{\mathrm{VG}} \sum_{i=1}^{12} S_{V i}
$$

where, $\lambda_{\mathrm{VG}}$ is coupling constant

$$
\begin{aligned}
& S_{\mathrm{VG}}=\lambda_{\mathrm{VG}} \int d^{4} x \sqrt{-g} \epsilon^{\alpha \gamma \nu} \epsilon^{\mu \eta \beta} \nabla_{\alpha \beta} A_{\gamma} \nabla_{\mu \nu} A_{\eta}, \\
& S_{V 1}=E_{1} \int d^{4} x \sqrt{-g} g^{\mu \nu} g^{\alpha \beta} g^{\gamma \delta} R_{\mu \nu} \nabla_{\alpha} A_{\gamma} \nabla_{\beta} A_{\delta}, \\
& S_{V 2}=E_{2} \int d^{4} x \sqrt{-g} g^{\mu \alpha} g^{\nu \beta} g^{\gamma \delta} R_{\mu \nu} \nabla_{\alpha} A_{\gamma} \nabla_{\beta} A_{\delta}, \\
& S_{V 3}=E_{3} \int d^{4} x \sqrt{-g} g^{\mu \nu} g^{\alpha \beta} g^{\gamma \delta} R_{\mu \nu} \nabla_{\alpha} A_{\beta} \nabla_{\gamma} A_{\delta}, \\
& S_{V 4}=E_{4} \int d^{4} x \sqrt{-g} g^{\mu \nu} g^{\alpha \delta} g^{\gamma \beta} R_{\mu \nu} \nabla_{\alpha} A_{\beta} \nabla_{\gamma} A_{\delta}, \\
& S_{V 5}=E_{5} \int d^{4} x \sqrt{-g} g^{\mu \gamma} g^{\alpha \beta} g^{\nu \delta} R_{\mu \nu} \nabla_{\alpha} A_{\beta} \nabla_{\gamma} A_{\delta}, \\
& S_{V 6}=E_{6} \int d^{4} x \sqrt{-g} g^{\mu \alpha} g^{\nu \delta} g^{\gamma \beta} R_{\mu \nu} \nabla_{\alpha} A_{\beta} \nabla_{\gamma} A_{\delta},
\end{aligned}
$$




$$
\begin{aligned}
& S_{V 7}=E_{7} \int d^{4} x \sqrt{-g} g^{\mu \alpha} g^{\nu \beta} g^{\gamma \zeta} g^{\delta \eta} R_{\alpha \beta \gamma \delta} \nabla_{\mu} A_{\nu} \nabla_{\zeta} A_{\eta}, \\
& S_{V 8}=E_{8} \int d^{4} x \sqrt{-g} g^{\mu \alpha} g^{\eta \beta} g^{\gamma \zeta} g^{\delta \nu} R_{\alpha \beta \gamma \delta} \nabla_{\mu} A_{\nu} \nabla_{\zeta} A_{\eta}, \\
& S_{V 9}=E_{9} \int d^{4} x \sqrt{-g} g^{\alpha \beta} g^{\gamma \delta} g^{\mu \nu} R_{\alpha \beta} R_{\gamma \delta} A_{\mu} A_{\nu},
\end{aligned}
$$

$$
\begin{aligned}
& S_{V 10}=E_{10} \int d^{4} x \sqrt{-g} g^{\alpha \beta} g^{\gamma \mu} g^{\delta \nu} R_{\alpha \beta} R_{\gamma \delta} A_{\mu} A_{\nu}, \\
& S_{V 11}=E_{11} \int d^{4} x \sqrt{-g} g^{\alpha \gamma} g^{\beta \delta} g^{\mu \nu} R_{\alpha \beta} R_{\gamma \delta} A_{\mu} A_{\nu}, \\
& S_{V 12}=E_{12} \int d^{4} x \sqrt{-g} g^{\alpha \gamma} g^{\beta \mu} g^{\delta \nu} R_{\alpha \beta} R_{\gamma \delta} A_{\mu} A_{\nu} .
\end{aligned}
$$

where $E_{i}$ 's are dimensionless coefficients. Note that $E_{i}$ are related to $\lambda_{\mathrm{VG}}$.
[1] B. Podolsky and P. Schwed, Rev. Mod. Phys. 20, 40 (1948).

[2] W. Thirring, Phys. Rev. 79, 703 (1950).

[3] A. Pais and G. E. Uhlenbeck, Phys. Rev. 79, 145 (1950).

[4] R. P. Woodard, Scholarpedia 10, 32243 (2015).

[5] J. Z. Simon, Phys. Rev. D 41, 3720 (1990).

[6] S. W. Hawking and T. Hertog, Phys. Rev. D 65, 103515 (2002).

[7] N. H. Barth and S. M. Christensen, Phys. Rev. D 28, 1876 (1983).

[8] K. S. Stelle, Phys. Rev. D 16, 953 (1977).

[9] E. S. Fradkin and A. A. Tseytlin, Nucl. Phys. B201, 469 (1982).

[10] T. P. Sotiriou and V. Faraoni, Rev. Mod. Phys. 82, 451 (2010).

[11] S. Capozziello and M. De Laurentis, Phys. Rep. 509, 167 (2011).

[12] S. Nojiri and S. D. Odintsov, Phys. Rep. 505, 59 (2011).

[13] T. Clifton, P. G. Ferreira, A. Padilla, and C. Skordis, Phys. Rep. 513, 1 (2012).

[14] S. Nojiri, S. D. Odintsov, and V. K. Oikonomou, Phys. Rep. 692, 1 (2017).

[15] M. Ishak, Living Rev. Relativity 22, 1 (2019).

[16] R. P. Woodard, Lect. Notes Phys. 720, 403 (2007).
[17] G. W. Horndeski, Int. J. Theor. Phys. 10, 363 (1974).

[18] C. Deffayet, S. Deser, and G. Esposito-Farese, Phys. Rev. D 80, 064015 (2009).

[19] C. Deffayet, A. E. Gümrükçüoğlu, S. Mukohyama, and Y. Wang, J. High Energy Phys. 04 (2014) 082.

[20] D. Lovelock, J. Math. Phys. (N.Y.) 12, 498 (1971).

[21] D. Lovelock, J. Math. Phys. (N.Y.) 13, 874 (1972).

[22] B. Zumino, Phys. Rep. 137, 109 (1986).

[23] T. Padmanabhan and D. Kothawala, Phys. Rep. 531, 115 (2013).

[24] D. Nandi and S. Shankaranarayanan, J. Cosmol. Astropart. Phys. 01 (2018) 039.

[25] L. Heisenberg, R. Kase, and S. Tsujikawa, Phys. Rev. D 98 , 024038 (2018).

[26] A. Nicolis, R. Rattazzi, and E. Trincherini, Phys. Rev. D 79, 064036 (2009).

[27] C. Deffayet, G. Esposito-Farese, and A. Vikman, Phys. Rev. D 79, 084003 (2009).

[28] G. Goon, K. Hinterbichler, A. Joyce, and M. Trodden, Phys. Lett. B 714, 115 (2012).

[29] K. Peeters, Comput. Phys. Commun. 176, 550 (2007).

[30] K. Peeters, arXiv:hep-th/0701238. 